\title{
1 Longitudinal immune cell profiling in early systemic lupus erythematosus
}

2 Takanori Sasaki ${ }^{1}$, Sabrina Bracero ${ }^{1}$, Joshua Keegan ${ }^{1}$, Lin Chen ${ }^{1}$, Ye Cao ${ }^{1}$, Emma

3 Stevens ${ }^{1}$, Yujie $\mathrm{Qu}^{2}$, Guoxing Wang ${ }^{2}$, Jennifer Nguyen ${ }^{1}$, Stephen E. Alves ${ }^{2}$, James

4 A. Lederer ${ }^{1}$, Karen H. Costenbader ${ }^{1}$, Deepak A. Rao ${ }^{1 *}$

$6{ }^{1}$ Brigham and Women's Hospital and Harvard Medical School, Boston, MA, USA

$7 \quad{ }^{2}$ Merck Sharp \& Dohme Corp., a subsidiary of Merck \& Co., Inc., Kenilworth, NJ,

8 USA

$10{ }^{*}$ Corresponding author

11 Deepak A. Rao

12 Brigham and Women's Hospital

13 Division of Rheumatology, Inflammation, and Immunity

14 Hale Building for Transformative Medicine, Room 6002

1560 Fenwood Road

16 Boston MA 02115

17 darao@bwh.harvard.edu

$18 \quad 617-525-1101$ 


\section{Abstract}

22 Objective: To investigate the immune cell profiling and their longitudinal changes in

23 systemic lupus erythematosus (SLE).

24 Methods: We employed mass cytometry with three different 38-39 marker panels

25 (Immunophenotyping, T cell/monocyte, and B cell) in cryopreserved peripheral

26 blood mononuclear cells (PBMCs) from nine patients with early SLE, 15 patients

27 with established SLE, and 14 non-inflammatory controls. We used machine

28 learning-driven clustering, FlowSOM (Flow Self-Organizing Maps) and dimensional

29 reduction with tSNE (t-distributed Stochastic Neighbor Embedding) to identify

30 unique cell populations in early and established SLE. For the nine early SLE patients,

31 longitudinal mass cytometry analysis was applied to PBMCs at three time points (at

32 enrollment, six months post-enrollment, and one year post-enrollment). Serum

33 samples were also assayed for 65 cytokines by Luminex multiplex assay, and

34 associations between cell types and cytokines/chemokines assessed.

35 Results: T peripheral helper cells (Tph cells), T follicular helper cells (Tfh cells) and

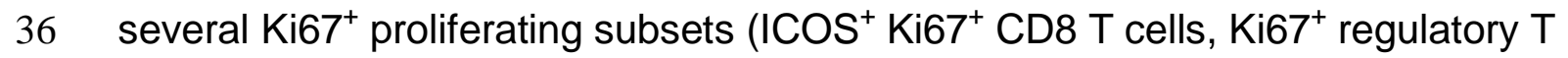

37 cells, CD19 ${ }^{\text {int }} \mathrm{Ki} 67^{\text {hi }}$ plasmablasts, and Ki67 ${ }^{\text {hi }}$ PU. $1^{\text {hi }}$ monocytes) were increased in

38 early SLE. Longitudinal mass cytometry and multiplex serum cytokine assays of

39 samples from early SLE patients revealed that Tfh cells and CXCL10 decreased at

40 one year post-enrollment. CXCL13 correlated positively with several of the

41 expanded cell populations in early SLE.

42 Conclusions: Two major helper T cell subsets and unique Ki67 ${ }^{+}$proliferating

43 immune cell subsets were expanded in the early phase of SLE, and the immunologic

44 features characteristic of early SLE evolved over time. 
Systemic lupus erythematosus (SLE) is a prototypical autoimmune disease that affects multiple vital organs. Untreated immune activation in SLE can lead to

47 tissue inflammation and irreversible organ damage, thus rapid recognition of lupus disease activity is an important goal in the care of patients with SLE. Delays in treatment are associated with poorer treatment responses and worse outcomes $(1-3)$.

Despite the importance of early recognition and intervention, diagnosis of early SLE is often difficult because initial manifestations of the disease frequently

53 include relatively non-specific symptoms. Fever, autoantibody production,

54 hypocomplementemia and leukopenia are relatively common in early SLE (4),

55 indicating that systemic immunologic features are already altered in the early phase.

56 We hypothesize that defining the alterations in immune cell populations early in

57 disease will provide critical insights into the early evolution of pathologic immune cell

58 activation in SLE and may yield key metrics to diagnose SLE in the early phase.

A series of single-cell RNA sequencing studies from inflamed tissues recently identified aberrant immune cell expansions and cytokine/chemokine-mediated

61 cellular networks within the affected organs in $\operatorname{SLE}(5,6)$. These unbiased analyses

62 provided broad and robust information on the composition of the immune cell

63 infiltrates in the kidney in lupus nephritis. However, since multiple biopsies are

64 difficult in most cases and most of the tissue samples were obtained from patients

65 with established disease, there is limited information on immunologic features early

66 in disease and little description of changes in inflammatory features between early

67 and later established phases of disease. This information is important for the

68 definition of immune response evolution over time in lupus, which may provide

69 insights into differences in treatment response over time. From this perspective, 
70 blood samples are easier to access and analyse longitudinally, yet few longitudinal

71 studies of associations between immunophenotype and clinical features in SLE over

72 time have been reported $(7,8)$.

73 Mass cytometry (or Cytometry by Time Of Flight, CyTOF) is a powerful tool to

74 broadly assess surface markers as well as intracellular proteins on immune cells.

75 Dimensional reduction and visualization with tSNE (t-distributed Stochastic

76 Neighbor Embedding) (9) combined with machine learning-driven clustering with

77 methods such as FlowSOM (Flow Self-Organizing Maps) (10) allow for

78 discrimination of distinct immune cell clusters in an unbiased way. Previously, the

79 increase of PD $-1^{\text {hi }}$ CXCR5 ${ }^{-}$CCR2 ${ }^{+}$CD4 T cells (T peripheral helper cells; Tph cells)

80 in the peripheral blood of patients with SLE was identified by this methodology (11).

81 CCR2 is a homing protein that promotes migration of immune cells to inflammatory

82 sites, suggesting that the increase of the circulating immune cell subset reflects an

83 inflammatory condition in the affected sites. Two studies have recently reported

84 analyses using mass cytometry to examine blood samples from patients with

85 established SLE $(12,13)$, but the longitudinal changes have not yet been studied.

86 Here we report broad mass cytometry data analyses with three different

87 38-39 marker panels in blood cells from patients with a new diagnosis of SLE. We

88 first identified several unique immune cell populations in early SLE through

89 unsupervised clustering and then verified the frequencies of these immune cell

90 subsets. We further investigated the immune cell frequencies and serum cytokine

91 levels in early SLE over time (at enrollment, six months, and 12 months post-

92 enrollment). These longitudinal analyses indicated that several unique $\mathrm{Ki} 67^{+}$

93 proliferating immune cell subsets are expanded even in the early phase of SLE and

94 remain consistently elevated over time. In contrast, T follicular helper cells (Tfh cells) 
95 appeared elevated early after diagnosis and decreased over time. Serum cytokine

96 profiling identified CXCL10, CD40L, IL-20, and TWEAK increased in early SLE, but

97 among them, CXCL10 decreased longitudinally. Our data provide a detailed

98 assessment of the immunologic features characteristic of early SLE as well as their

99 changes over time.

100

101 Results

102 An unsupervised cell clustering view of the immune cell landscape in SLE.

103 To investigate immunological and longitudinal changes in SLE, we evaluated

104 cross-sectional and longitudinal analyses of peripheral blood mononuclear cells

105 (PBMCs) by mass cytometry using three different panels (broad immunophenotype

106 panel, T cell/monocyte-focused panel, and B cell-focused panel), along with

107 65-analyte serum cytokine profiling data (Figure 1A, S-Table 1). As an overview of

108 our approach, we first applied unsupervised cell clustering using FlowSOM and

109 dimensionality reduction by tSNE to the cross-sectional mass cytometry data from

110 nine patients with early SLE who were enrolled within six months after the diagnosis,

11115 patients with established SLE, and 14 non-inflammatory controls to identify

112 distinct populations in an unbiased way. Early SLE patients were younger than

113 established SLE patients (21.6 vs 36.5 years old, $\mathrm{P}<0.001$ ), and corticosteroid (CS)

114 use (33.3 vs 93.3\%, $P=0.03$ ) and the dose (2.5 vs $14.5 \mathrm{mg} /$ day, $\mathrm{P}=0.01$ ) were higher

115 in established SLE patients. SLEDAI-2K disease activity were comparable between

116 the two groups (5.8 vs 5.1, $\mathrm{P}=0.80)(\mathrm{S}-\mathrm{Table} 2,3)$. We then investigated the

117 longitudinal changes of the distinct immune cell populations and 65 cytokine levels

118 from the nine patients with early SLE (Figure 1B), at three time points $(A=$ at

119 enrollment, $\mathrm{B}=$ six months after the enrollment, $\mathrm{C}=12$ months after the enrollment). 
120 Finally, we analysed associations between cell types and cytokines by hierarchical

121 clustering.

122 For an initial, high-level view of the circulating immune cell populations, we

123 first performed tSNE clustering of Immunophenotype panel mass cytometry data

124 from the cross-sectional cohorts (Figure 1C). tSNE allowed clear visualization of

125 distinct immune cell clusters, including three major $\mathrm{CD}^{+} \mathrm{T}$ cell populations, $\mathrm{CD}^{-}$

$126 \mathrm{CD}{ }^{+}$NK cells, CD19 ${ }^{+}$B cells, CD14 ${ }^{+}$monocytes, CD14 CD11c ${ }^{+}$myeloid dendritic

127 cells (mDCs). Two of the $\mathrm{CD}^{+}$clusters were $\mathrm{CD} 3^{+} \mathrm{CD}^{+}{ }^{\mathrm{T}}$ cells and $\mathrm{CD} 3^{+} \mathrm{CD}^{+} \mathrm{T}$

128 cells. A third cluster, CD3 ${ }^{+}$CD4 $^{-}$CD8 $^{-}$T cells expressed TCR $\gamma \delta$, identifying this

129 population as $\gamma \delta$ T cells. Among these high-level immune cell subsets, the proportion

130 of $\mathrm{CD}^{+} \mathrm{CD}^{+} \mathrm{T}$ cells was increased and the proportion of $\mathrm{CD}^{+} \mathrm{CD}^{+} \mathrm{T}$ cells was

131 decreased in established SLE patients compared to controls, but none of these

132 populations were higher in early SLE compared to controls.

134 Expanded $\mathrm{Ki}^{+}$activated CD8 T cells in SLE patients.

135 We next investigated changes in CD8 T cell populations in early SLE. To

136 identify cell populations that differ between controls and SLE patients, we clustered

137 CD8 T cells based on the 39-marker T cell-focused panel using FlowSOM. We

138 compared the abundances of the clusters between SLE and controls and identified

139 metacluster 10 as significantly increased in early SLE patients (5.2-fold, $\mathrm{P}<0.05$,

140 Kruskal-Wallis with Dunn's multiple comparisons test) (Figure 2A). Heatmap

141 expression analysis revealed that metacluster 10 contained cells with high

142 expression of Ki67 and ICOS, suggesting that they are proliferating CD8 T cells

143 (Figure 2B). Metaclusters 5 and 9 showed a similar expression pattern to

144 metacluster 10 with high expression of Ki67 and ICOS and tended to be higher in 
145 SLE (Figure 2A). tSNE visualization of merged data from early SLE patients

146 confirmed that the $\mathrm{Ki} 67^{+}$proliferative population expressed ICOS (Figure 2C). This

147 population also expressed PD-1 and HLA-DR, suggesting that these are activated

148 CD8 T cells. Interestingly, the Ki67 ${ }^{+}$CD8 T cells did not highly express granzyme B

149 and granzyme K. Biaxial plots demonstrated that Ki67 ${ }^{+} \mathrm{ICOS}{ }^{+} \mathrm{CD} 8 \mathrm{~T}$ cells

150 significantly increased in early SLE compared to controls ( 0.8 vs $3.5 \%, P<0.01$,

151 Kruskal-Wallis with Dunn's multiple comparisons test) (Figure 2D).

152 In longitudinal analyses including time points six and 12 months

153 post-enrollment, $\mathrm{Ki} 67^{+} \mathrm{ICOS}^{+} \mathrm{CD} 8 \mathrm{~T}$ cells remained persistently elevated over time.

154 Disease activity in this patient cohort remained similarly active during this time frame

155 (Figure 2E, F). We also identified metacluster 13, which contained T cells with high

156 expression of CD94, CD56, and TCRV $\delta 2$, as significantly decreased in early SLE

157 and established SLE patients (Figure 2A, B). Metacluster 3, which contained CD96 ${ }^{+}$

158 CD8 T cells, was reduced in established SLE, but not in early SLE (Figure 2A, B).

Tfh cells but not Tph cells decreased over time in early SLE.

We next applied FlowSOM to CD4 T cells in controls and SLE patients. We

162 identified metaclusters 6 (2.3-fold, $\mathrm{P}<0.01$ ), 13 (5.8-fold, $\mathrm{P}<0.01$ ), 14 (2.8-fold,

$163 \mathrm{P}<0.05)$, and 15 (3.9-fold, $\mathrm{P}<0.01)$ significantly increased in early SLE patients at

164 diagnosis (Figure 3A). Cells in metacluster 6 and metacluster 13 highly expressed

165 PD-1, ICOS, and CD40L, but lacked CXCR5, suggesting that these two

166 metaclusters contained Tph cells (Figure 3B). Interestingly, these two clusters were

167 quite different in expression of CXCR3 and T-bet; low expression in metacluster 6

168 and high expression in metacluster 13. Metacluster 14 could be classified as Tfh

169 cells with the high expression of PD-1, ICOS, and CXCR5. Metacluster 15 
170 demonstrated a proliferating Treg phenotype with high expression of Ki67, FoxP3,

171 CTLA-4, Helios, CD25, and CD39, and low expression of CD127 (Figure 3B). tSNE

172 visualization revealed distinct clusters of PD-1 in either $\mathrm{CXCR} 5^{+}$or $\mathrm{CXCR}^{-}$regions

173 and $\mathrm{Ki} 7^{+}$FoxP3-expressing Treg in early SLE patients (Figure 3C).

174 Quantification by biaxial gating confirmed that CXCR5 PD-1 ${ }^{\text {hi }}$ Tph cells

175 (controls: 3.0\%, early SLE: 7.0\%, established SLE: 8.9\%, P<0.05 in controls vs early

176 SLE, $\mathrm{P}<0.01$ in controls vs established SLE), CXCR5 ${ }^{+} \mathrm{PD}-1^{\text {hi }}$ Tfh cells (controls:

$1772.0 \%$, early SLE: 6.2\%, established SLE: 4.4\%, $\mathrm{P}<0.001$ in controls vs early SLE,

$178 \mathrm{P}<0.05$ in controls vs established SLE), and $\mathrm{Ki}^{+} 7^{+} \mathrm{FoxP}^{+}$Treg cells (controls: $1.0 \%$,

179 early SLE: $3.6 \%$, established SLE: $2.5 \%, P<0.01$ in controls vs early $S L E, P<0.01$ in

180 controls vs established SLE) were increased in both early SLE and established SLE

181 patients (Figure 3D, E). We also found that Tph cells and Ki67 ${ }^{+}$Treg cells were

182 consistently elevated at one year after the diagnosis, whereas Tfh cells decreased

183 over time in the early SLE cohort (Figure 3F). The proportion of Tfh cells did not

184 correlate with corticosteroid dose and was not significantly different between the

185 patients treated with prednisone $>10 \mathrm{mg}$ and/or mycophenolate mofetil (MMF) and

186 those without, suggesting that the decrease is independent from

187 immunosuppressive treatment (Figure 3G). We identified metaclusters 1 and 3

188 significantly decreased in established SLE patients (Figure 3A). These metaclusters

189 highly expressed CD127 and CD40L, but CXCR5 was quite distinct between

190 metacluster 1 (CXCR5 $)$ and metacluster 3 (CXCR5 $\left.{ }^{+}\right)$(Figure 3B).

192 Increased CD19 ${ }^{\text {int }} \mathrm{Ki}^{\mathrm{hi}}{ }^{\text {hi }}$ plasmablasts in early SLE.

193 We next applied the same clustering approach to CD19 B cells, now using

194 the B cell-focused mass cytometry panel (S-Table 1). We found metacluster 4 
195 increased in early SLE patients (4.7-fold, $P<0.05)$, metaclusters 11, 12, 13, and 15

196 increased in established SLE patients, and metacluster 14 increased in both early

197 SLE (3.0-fold, P<0.05) and established SLE (Figure 4A). Expression heatmap

198 analysis indicated that metacluster 4 contained a CD19 ${ }^{\text {int }}$ Ki67 ${ }^{\text {hi }}$ population with high

199 expression of CD27 and CD38, indicating proliferating plasmablasts (Figure 4B).

200 We also identified 5 metaclusters consistent with CD11c ${ }^{+}$T-bet $^{+}$CD21 $1^{\text {low }}$ CXCR5 $^{-}$

201 age-associated $B$ cells (ABCs): HLA-DR ${ }^{+} \mathrm{CD}^{-} 8^{-} \operatorname{lgG}^{+}$ABCs (metacluster 11),

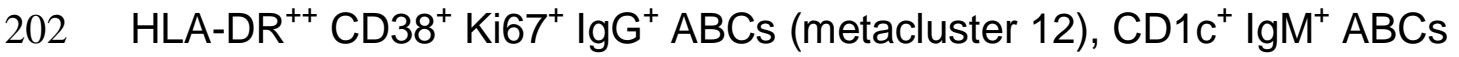

203 (metacluster 13), $\operatorname{lgM}^{+} \lg ^{+}$ABCs (metacluster 14), and CD11c ${ }^{\text {hi }}$ T-bet $^{\text {hi }}$ ABCs

204 (metacluster 15). tSNE visualization demonstrated distinct marker expression of

205 CD19 $^{\text {int }} \mathrm{Ki}^{\text {hi }}{ }^{\text {plasmablasts and CD11c }}$ T-bet $^{+}$CD2 $^{\text {low }}$ CXCR5 $^{-}$ABCs in early SLE

206 (Figure 4C). Biaxial plots confirmed that the CD19 ${ }^{\text {int }} \mathrm{Ki67} 7^{\text {hi }}$ population contained

$207 \mathrm{CD}^{+} 7^{+} \mathrm{CD}^{+} 8^{+}$plasmablasts, but CD19 ${ }^{\text {hi }} \mathrm{Ki6} 7^{\text {low }}$ population did not (Figure 4D).

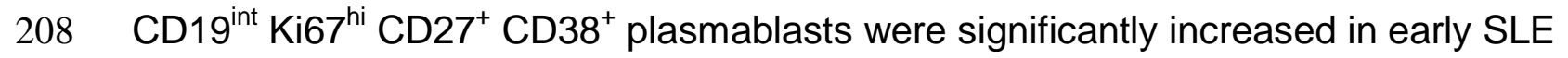

209 patients (controls: $0.07 \%$, early SLE: 0.88\%, established SLE: $0.18 \%, P<0.01$ in

210 controls vs early SLE), whereas CD11C ${ }^{+}$CD21 ${ }^{\text {low }}$ ABCs were more expanded in

211 established SLE patients (controls: 3.8\%, early SLE: 7.6\%, established SLE: 14.8\%,

$212 \mathrm{P}<0.001$ in controls vs established SLE) (Figure 4D, E). CD19 ${ }^{\text {int }} \mathrm{Ki67}^{\mathrm{hi}} \mathrm{CD} 27^{+}$

213 CD38 $^{+}$plasmablasts were significantly lower in established SLE compared to early

214 SLE patients, but also significantly lower in the SLE patients treated with prednisone

$215>10 \mathrm{mg}$ and/or MMF compared the others $(0.14 \%$ vs $1.1 \%, \mathrm{P}<0.01)$ (S-Figure 1),

216 suggesting that treatment may affect plasmablast abundance in treated SLE

217 patients. Consistent with the increased abundance of metacluster 14 in early SLE,

$218 \operatorname{lgM}^{+} \lg \mathrm{D}^{+} \mathrm{ABCs}$ were significantly higher in early SLE patients compared to controls

219 (Figure 4F). For other subclasses, almost $40 \%$ of CD19 ${ }^{\text {int }} \mathrm{Ki}^{\mathrm{hi}} \mathrm{CD}^{\mathrm{hi}} \mathrm{C}^{+} \mathrm{CD}^{+} 8^{+}$ 
220 plasmablasts were $\lg A$, and $\lg G$ was rare, whereas $\lg G$ was more frequent $(20 \%)$ in

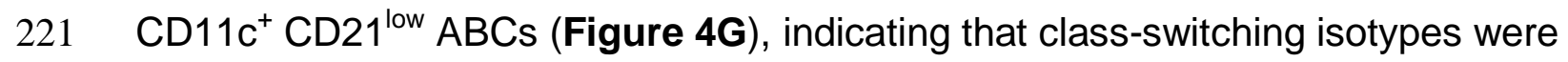

222 different in $\mathrm{CD}_{19}{ }^{\text {int }} \mathrm{Ki} 7^{\text {hi }} \mathrm{CD} 27^{+} \mathrm{CD} 8^{+}$plasmablasts and CD11c ${ }^{+} \mathrm{CD} 21^{\text {low }} \mathrm{ABCs}$. In

223 longitudinal analyses, $\mathrm{CD} 19^{\mathrm{int}} \mathrm{Ki} 67^{\mathrm{hi}} \mathrm{CD} 27^{+} \mathrm{CD} 38^{+}$plasmablasts and $\mathrm{ABC}$, and $\lg \mathrm{G}$

224 or IgA class-switched CD19 ${ }^{\text {int }} \mathrm{Ki} 67^{\text {hi }} \mathrm{CD} 7^{+} \mathrm{CD} 8^{+}$plasmablasts and ABCs, stayed

225 at high levels at one year after enrollment (S-Figure 2, Figure 4H). We also

226 identified metacluster 6 , which contained $\lg A^{+} \lg D^{-} \mathrm{CD}^{2} 7^{+}$memory B cells, as

227 relatively decreased in early SLE patients.

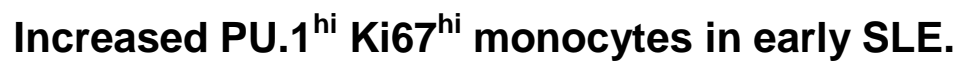

230 In the CD14 ${ }^{+}$monocyte FlowSOM analysis using the T cell/monocyte panel,

231 metacluster 8 was decreased and metacluster 13 was increased in early (5.6-fold,

$232 \mathrm{P}<0.05)$ and established SLE patients compared to controls (Figure 5A). Heatmap

233 analysis indicated that metacluster 13 contained HLA-DR- PU. $1^{\text {hi }} \mathrm{Ki}^{\mathrm{h}} 7^{\text {hi }}$ monocytes

234 (Figure 5B). tSNE visualization confirmed that $\mathrm{Ki} 67^{\mathrm{hi}}$ monocytes highly expressed

235 PU.1 but not HLA-DR (Figure 5C). Biaxial plots revealed that metacluster 13 was

236 well correlated with $\mathrm{HLA}^{-D R^{-}} \mathrm{Ki}^{\mathrm{hi}} 7^{\text {hi }}$ monocytes and PU.1 ${ }^{\text {hi }}$ Ki67 ${ }^{\text {hi }}$ monocytes but

237 more strongly in PU. $1^{\text {hi }}$ Ki67 ${ }^{\text {hi }}$ monocytes (Figure 5D). Consistent with the FlowSOM

238 analysis, PU. $1^{\text {hi }}$ Ki67 ${ }^{\text {hi }}$ monocytes were increased in patients with early SLE, with

239 comparable levels to established SLE patients (controls: 7.6\%, early SLE: 29.6\%,

240 established SLE: 29.7\%, P<0.05 in controls vs early SLE, $\mathrm{P}<0.05$ in controls vs

241 established SLE) (Figure 5E). PU.1 ${ }^{\text {hi }}$ Ki67 $7^{\text {hi }}$ monocytes expressed higher levels of

242 CCR2 compared to PU. $1^{\text {low }}$ Ki67 $7^{\text {low }}$ monocytes $(\mathrm{P}<0.001)$ (Figure 5F). PU.1 ${ }^{\text {hi }}$ Ki67 ${ }^{\text {hi }}$

243 monocyte frequency did not change over time in early SLE patients (Figire 5G). 


\section{Associations between expanded immune cell populations in SLE.}

246 Since the analyses across multiple mass cytometry panels revealed that 247 several $\mathrm{Ki} \mathrm{7}^{+}$proliferating populations were expanded in patients with SLE, we 248 hypothesized that $\mathrm{Ki} 67^{+} \mathrm{NK}$ cells would also be increased in SLE. As we expected, 249 biaxial plots indicated that $\mathrm{NKG}^{2} \mathrm{D}^{+} \mathrm{Ki}^{2} 7^{+} \mathrm{CD}^{-} \mathrm{CD}^{2} 6^{+} \mathrm{NK}$ cells were highly

250 increased in SLE, with stable levels in patients with early SLE over time (Figure 6A, 251 B). The Ki67 ${ }^{+}$population did not express PD-1, HLA-DR, and ICOS, unlike Ki67 ${ }^{+}$ 252 CD4 or CD8 T cells (Figure 6C). Next, to identify associations between expanded 253 immune cell populations in early SLE, we applied a hierarchical clustering analysis 254 using the frequencies of $\mathrm{Ki}^{+} 7^{+} \mathrm{ICOS}^{+} \mathrm{CD} 8 \mathrm{~T}$ cells, Tph cells, Tfh cells, IgG ${ }^{+} \mathrm{CD} 19^{\text {int }}$

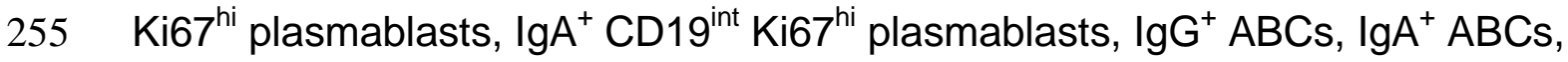

256 PU. $1^{\text {hi }}$ Ki67 ${ }^{\text {hi }}$ monocytes, and NKG2D+ Ki67 ${ }^{+}$NK cells. This analysis segregated cell 257 populations into clusters with distinct patterns, including one cluster of PU. ${ }^{\text {hi }} \mathrm{Ki} 67^{\text {hi }}$ 258 monocytes and NKG2D ${ }^{+} \mathrm{Ki} 67^{+}$NK cells (innate immunity cluster), one cluster of $259 \mathrm{Ki}^{+} \mathrm{ICOS}^{+} \mathrm{CD} 8 \mathrm{~T}$ cells, Tph cells, and Tfh cells ( $\mathrm{T}$ cell cluster), and one larger 260 cluster of $\operatorname{lgG}^{+} \mathrm{CD} 19^{\text {int }} \mathrm{Ki} 7^{\text {hi }}$ plasmablasts, $\lg \mathrm{A}^{+} \mathrm{CD} 19^{\text {int }} \mathrm{Ki} 67^{\text {hi }}$ plasmablasts, $\operatorname{lgG}^{+}$

$261 \mathrm{ABCs}$, and IgA $\mathrm{A}^{+} \mathrm{ABC}$ (B cell cluster) (Figure 6D). Notably, Tph cells correlated with $262 \operatorname{ABCs}(\rho=0.51 P=0.006)$ and $C D 19^{\text {int }} K i 67^{\text {hi }}$ plasmablast $(\rho=0.43 P=0.01)$, whereas 263 Tfh cells did not (Figure 6E).

Longitudinal cytokine and chemokine profiling in early SLE.

267 controls and nine early SLE patients, with the early SLE patients again analysed at 268 three timepoints as in the cytometry analyses. Among the 65 cytokines/chemokines 269 we selected a priori to be potentially important in early SLE pathogenesis, 33 
270 cytokines were detected in serum samples. Interestingly, these cytokines positively

271 correlated with each other together, suggesting a co-ordinately regulated underlying

272 cytokine network in early SLE (Figure 7A). Most cytokines, with the exception of

273 IL-16, had higher levels in early SLE samples, and IL-2R, CXCL10, CXCL13,

274 IL-12p70, IL-17A, TSLP, CCL8, CCL24, TNF-RII, IL-2, IL-20, CD40L, CCL3, CD30,

275 and TWEAK were significantly increased in early SLE samples, and CXCL10,

276 CD40L, IL-20, and TWEAK remaining significantly higher even after Bonferroni

277 correction to adjust for multiple testing (Figure 7B, S-Figure 3, S-Figure 4). Among

278 these four cytokines, CXCL10 (P=0.03) was significantly decreased at 1 year after

279 diagnosis, but CD40L, IL-20, and TWEAK stayed high levels. (Figure 7C, S-Figure

280 5). Next, to clarify the potential association between immune cells and chemokines,

281 we investigated correlations between immune cell frequencies and serum

282 chemokine levels in early SLE. Notably, CXCL13 broadly and strongly correlated

283 with expanded lymphocyte subsets (Tph, Tfh, Ki67 ${ }^{\text {hi }}$ COS $^{+}$CD8, ABC,

284 plasmablasts) in samples from patients with SLE (Figure 7D). In contrast, CCL2 was

285 strongly correlated with PU. $1^{\text {hi }} \mathrm{Ki} 67^{\text {hi }}$ monocytes, the subset that highly expressed

286 CCR2, suggesting the involvement of CCL2-CCR2 axis for PU. $1^{\text {hi }}$ Ki67 $7^{\text {hi }}$ monocytes

287 migration to inflamed sites. These results suggest that different co-regulated

288 pathways, which link cell types to related circulating factors, are active in early SLE

289 patients.

291 Discussion

By broad and longitudinal cellular immunophenotyping and serum

293 cytokine/chemokine profiling, we identified multiple expanded immune cell

294 populations in patients with early SLE and evaluated their changes in the first year of 
295 disease and their relationships with serum cytokines/chemokines. We found that

296 several lymphocyte populations expanded in early SLE share a common feature of

297 expression of Ki67, a well-established marker of lymphocyte proliferation. This

298 shared cytometric feature may capture the broad, active immune response occurring

299 in early SLE. These Ki67 $7^{+}$populations, as well as Tph cells and ABCs, remain

300 consistently elevated over the first year and are similarly elevated in established SLE

301 patients, suggesting that these pathways are activated early and continue to

302 characterize the pathologic immune response in SLE.

303 Notably, we also identified specific features of the immune response that

304 change over time in early SLE patients. In particular, Tfh cells decreased over time in

305 early SLE patients. Although Tfh cells and some of their inducing factors, such as

306 IL-12, have been considered as therapeutic targets in SLE $(14,15)$, a phase III study

307 of ustekinumab, a monoclonal antibody targeting interleukin IL-12 and IL-23, was

308 discontinued due to the lack of efficacy (LOTUS study; NCT03517722). Since Tfh

309 expanded initially, but decreased longitudinally, this target may have the therapeutic

310 "window of opportunity". Moreover, CD40L, IL-20, and TWEAK were increased at

311 the initial time point and persistently elevated, while CXCL10 decreased over time.

312 These data suggested that immune profiles change in each phase of SLE (Figure 8),

313 such that quantification of some features of the immune response in SLE need to be

314 adjusted based on disease duration.

315 Diagnosing early SLE is challenging because the initial clinical manifestations

316 are often non-specific. Identifying the immune system activation associated with

317 early SLE may help to diagnose SLE as early as possible. Our study revealed that

318 both antibody-secreting plasmablasts and B cell-helper T cells, including Tfh cells

319 and Tph cells, were activated in the early phase and could be markers for early SLE. 
320 Since autoantibodies are increased several years prior to SLE onset (16), but not all

321 autoantibody positive individuals develop SLE, it will be of major interest to

322 determine whether alterations in circulating activated B cells and B cell-helper T can

323 serve as specific hallmarks to predict risk of developing clinically evident SLE.

$324 \quad$ Both Tph cells and Tfh cells contribute to B cell responses through the

325 production of IL-21, CD40L, and CXCL13 $(17,18)$. Strikingly, Tph cells stayed at

326 high levels during the first year post-enrollment, whereas Tfh cells decreased

327 longitudinally, suggesting distinct roles for Tph cells and Th cells over the course of

328 the disease. One major difference between Tph cells and Tfh cells is their

329 chemokine receptor expression, which determines their migratory capacity. Tph

330 cells migrate into local inflammatory sites through receptors such as CCR2 and

331 CCR5, while Tfh cells accumulate in B cell follicles within secondary lymphoid

332 organs via a CXCR5-CXCL13 axis (19). Our data imply that Tfh-B cell interactions in

333 secondary lymphoid organs may be particularly important at the initial onset of SLE,

334 but the importance may shift to Tph-B cell interactions at local inflammatory sites

335 over time. We did collect detailed data on SLE therapies administered to the newly

336 diagnosed patients, but our small sample size precludes detailed analysis of how

337 these therapies may impact lymphocyte populations and cytokines/chemokines over

338 the first year of disease and this also deserves further study.

339 In the B cell analysis, the dominant subclasses differed in ABCs and CD19

$340 \mathrm{Ki} 67^{\text {hi }}$ plasmablasts. Recent broad BCR analysis of six different autoimmune

341 diseases indicated that plasmablasts expressed more $\lg A 1 / 2$ compared to $\lg \mathrm{G} 1 / 2$,

342 whereas IgD CD27 B cells, which contain much of the ABC population, expressed

343 more $\operatorname{lgG} 1 / 2$ (20). Among these six autoimmune diseases, the frequency of $\lg A 1 / 2$

344 in PBMC B cells was higher in SLE, IgA vasculitis, Crohn's disease, and Bechet's 
345 disease compared to healthy controls. Considering that the mucosal associated

346 lymphoid tissues (MALT) or gut-associated lymphoid tissues (GALT) are the main

347 source of $\lg A^{+}$plasmablasts (21), intestinal dysbiosis might be involved in the

348 increase of plasmablasts in SLE.

349 We found that an expanded monocyte population in SLE expressed elevated

350 levels of PU.1, a transcription factor implicated in macrophage development and

351 function (22). Previous single-cell RNA-seq analyses of kidney biopsy samples

352 suggested that inflammatory monocytes differentiate into phagocytic and M2-like

353 macrophages in lupus nephritis (5). As PU. $1^{\text {hi }} \mathrm{Ki} 67^{\text {hi }}$ monocytes expressed CCR2

354 and correlated well with serum CCL2, this monocyte population in the blood may be

355 a precursor of inflammatory monocytes that infiltrate tissues.

356 A hierarchical clustering of immune cell subsets revealed distinct clusters of

357 immune subsets with correlated abundance patterns, including clusters reflective of

358 innate immunity, T cell activation, and B cell activation. Of note, Tph cells and Ki67 ${ }^{+}$

$359 \mathrm{ICOS}^{+}$CD8 T cells were strongly correlated, suggesting that these two subsets may

360 be regulated through a common inducing factor. In this context, type I IFN may play

361 an important role in the regulation. A series of RNA-seq analyses indicated that IFN

362 signatures were highly enriched in Tph cells in SLE (11) and Ki67 ${ }^{\text {hi }}$ CD8 T cells in

363 immune checkpoint inhibitor-associated arthritis patients (23). In addition, type I IFN

364 has negative regulatory effects on the expression of CXCR5 $(24,25)$. As Tph cells

365 and $\mathrm{Ki}_{67}{ }^{+} \mathrm{ICOS}^{+} \mathrm{CD} 8 \mathrm{~T}$ cells may be pathogenic drivers of SLE, anifrolumab, a fully

366 human monoclonal antibody against the type I IFN receptor (26), may act to

367 ameliorate the disease activity in part through the regulation of Tph cells and Ki67

$368 \mathrm{ICOS}^{+} \mathrm{CD} 8 \mathrm{~T}$ cells. 
Our study has several limitations. The relatively small cohort of early SLE patients followed limits the ability to identify co-correlated immune features and

371 precludes evaluation of clinical correlates of the cellular features identified. A larger

372 cohort will be required in subsequent studies to determine the potential prognostic

373 significance of the immune features detected here. We have quantified cytometric

374 and serum protein features but have not interrogated transcriptional programs. In

375 addition, our study focuses only on blood samples and does not contain parallel

376 tissue studies. Nonetheless, the substantial alterations demonstrated in circulating

377 immune cells from patients with lupus support the idea that clinically relevant signals

378 may be detectable in blood samples.

379 In conclusion, this study highlighted persistent activation of Tph, ABCs, and

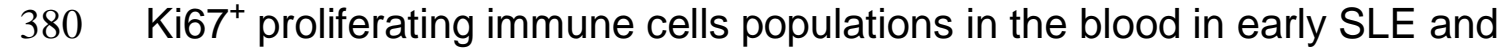

381 underscores the value of broad, longitudinal immunophenotyping to define patterns

382 of SLE immune activity that may help refine potential biomarkers and prioritize

383 therapeutic targets for early and established phases of SLE.

\section{Methods}

\section{Study Subjects}

All SLE patients met 1997 ACR classification criteria (27). For the early SLE

388 cohort, nine SLE patient were who were within six months of disease diagnosis and

389 without treatment with major immunosuppressive therapies (treatment with

390 prednisone $\leq 10 \mathrm{mg}$ and hydroxychloroquine were permitted). For the

391 cross-sectional study, 14 non-inflammatory controls and 15 patients with established

392 SLE were also included. For the longitudinal cytometry study, the same nine patients

393 with early SLE were evaluated at six months and 12 months after enrollment. For 
394 serum analyses, the same nine early SLE patients were evaluated, along with nine

395 non-inflammatory controls different from the cross-sectional study. Detailed clinical

396 information is shown in Supplementary Table 2, 3.

397

\section{Mass cytometry}

Blood samples were collected into heparin tubes and PBMCs were isolated

400 by density centrifugation using Ficoll-Hypaque in $50 \mathrm{~mL}$ conical tubes. PBMCs were 401 washed by PBS and cryopreserved in a 10\% DMSO + 90\% FBS solution. Samples

402 from the cross-sectional cohorts as well as longitudinal samples from the early SLE 403 cohort were collected and thawed together in batches of 20 samples per batch (total 404 three batches) and processed for mass cytometry within a one-week period. The 405 three longitudinal samples from each early SLE patient were included in the same 406 batch to minimize potential batch effects.

407 Cryopreserved PBMCs were thawed into RPMI Medium 1640 (Life 408 Technologies \#11875-085) supplemented with 5\% heat-inactivated fetal bovine 409 serum (Life Technologies \#16000044), 1 mM GlutaMAX (Life Technologies 410 \#35050079), antibiotic-antimycotic (Life Technologies \#15240062), 2 mM MEM 411 non-essential amino acids (Life Technologies \#11140050), 10 mM HEPES (Life

412 Technologies \#15630080), $2.5 \times 10^{-5}$ M 2-mercaptoethanol (Sigma-Aldrich \#M3148), 41320 units/mL sodium heparin (Sigma-Aldrich \#H3393), and 25 units/mL benzonase 414 nuclease (Sigma-Aldrich \#E1014). Cells were counted and $0.5-1 \times 10^{6}$ cells from 415 each sample were transferred to a polypropylene plate for staining. The samples 416 were spun down and aspirated. $5 \mu \mathrm{M}$ of cisplatin viability staining reagent (Fluidigm 417 \#201064) was added for two minutes and then diluted with culture media. After 418 centrifugation, Human TruStain FcX Fc receptor blocking reagent (BioLegend 
419 \#422302) was used at a 1:100 dilution in PBS with $2.5 \mathrm{~g}$ bovine serum albumin

420 (Sigma Aldrich \#A3059) and $100 \mathrm{mg}$ of sodium azide (Sigma Aldrich \#71289) for 10

421 minutes followed by incubation with conjugated surface antibodies for 30 minutes.

422 All antibodies were obtained from the Harvard Medical Area CyTOF Antibody

423 Resource and Core (Boston, MA).

$424 \quad 16 \%$ stock paraformaldehyde (Fisher Scientific \#04042-500) dissolved in

425 PBS was used at a final concentration of $4 \%$ formaldehyde for 10 minutes in order to

426 fix the samples before permeabilization with the FoxP3/Transcription Factor Staining

427 Buffer Set (ThermoFisher Scientific \#00-5523-00). The samples were incubated with

428 SCN-EDTA coupled palladium based barcoding reagents for 15 minutes and then

429 combined into a single sample. Conjugated intracellular antibodies were added into

430 each tube and incubated for 30 minutes. Cells were then fixed with $1.6 \%$

431 formaldehyde for 10 minutes. DNA was labelled for 20 minutes with an $18.75 \mu \mathrm{M}$

432 iridium intercalator solution (Fluidigm \#201192B). Samples were subsequently

433 washed and reconstituted in Milli-Q filtered distilled water in the presence of EQ Four

434 Element Calibration beads (Fluidigm \#201078) at a final concentration of $1 \times 10^{6}$

435 cells/mL. Samples were acquired on a Helios CyTOF Mass Cytometer (Fluidigm).

437 FlowSOM and tSNE analyses

438 The raw FCS files were normalized to reduce signal deviation between

439 samples over the course of multi-day batch acquisitions, utilizing the bead standard 440 normalization method established by Finck et al (28). These normalized files were

441 then deconvoluted into individual sample files using a single-cell based debarcoding

442 algorithm established by Zunder et al (29) Mass cytometry data were gated to

443 exclude debris and identify $\mathrm{DNA}^{+}$events. Non-viable cisplatin ${ }^{+}$cells and equalization 
444 beads were excluded. FlowSOM analyses were performed using Cytobank. HCs

$445(n=14)$ and SLE ( $n=24$ : early SLE $n=9$, established SLE $n=15)$ samples were

446 included. Metacluster and cluster numbers were 15 and 225, respectively. Each

447 metacluster abundances were compared between HCs, early SLE, and established

448 SLE. Heatmap analyses were performed with Z score of metacluster medians in

449 each marker. For tSNE clustering, HCs and SLE fcs files were concatenated using

450 FlowJo 10.4.2. tSNE analyses using Cytobank was performed with equal number

451 events from the concatenated HCs or SLE fcs files. Gating for cell frequencies and

452 expression intensity quantification were performed using FlowJo 10.4.2.

\section{Cytokines}

65 cytokines (Supplementary figure 1) were measured by Luminex multiplex

456 assay according to the manufacturer's instructions. Associations between cytokines,

457 and association cytokines and cell types assessed with heatmap analysis using

458 Spearman's correlation coefficient.

\section{Study approval}

461 SLE patients and non-inflammatory controls were enrolled at Brigham and

462 Women's Hospital with informed consent under IRB protocols (2014P002558 and

463 2016P001660) approved by Mass General Brigham IRB.

\section{Statistics}

Statistical comparisons were performed in Prism. Mann-Whitney test was

467 used for comparison between two groups, and Kruskal-Wallis with Dunn's multiple

468 comparisons test for comparisons between three groups. Wilcoxon test was used for 
469 comparison between time point $A$ and $C$ in the longitudinal analyses. Correlation

470 analysis was performed by Spearman's test, and the best fit line were drawn when

471 significant. Heatmap analysis was performed P-value $<0.05$ (two sided) was

472 regarded as significant. Data are shown as mean $\pm \mathrm{SE}$.

474 Data availability

475 Data are available upon reasonable request to the corresponding author.

\section{Acknowledgements}

478 This work is supported in part by funding from Merck Sharp \& Dohme Corp., a

479 subsidiary of Merck \& Co., Inc., Kenilworth, NJ, USA, and NIAMS K24 AR066109 to

$480 \mathrm{KHC}$ and from the Lupus Research Alliance, Burroughs Wellcome Fund Career

481 Award in Medical Sciences, Doris Duke Charitable Foundation Clinical Scientist

482 Development Award, NIAMS K08 AR072791 to DAR, and NIAMS P30 AR070253 to 483 JAL and DAR.

\section{Disclosures}

486 Dr Costenbader reports personal fees $(<\$ 10,000)$ and research support from Merck, 487 Amgen, Astra Zeneca, Eli Lilly, Exagen Diagnostics, Gilead, Glaxo Smith Kline, 488 Janssen and Neutrolis. Dr. Rao reports personal fees from Pfizer, Janssen, Merck, 489 Scipher Medicine, GlaxoSmithKline, and Bristol-Myers Squibb $(<\$ 10,000)$, grant 490 support from Merck related to the submitted work, and grant support from

491 Bristol-Myers Squib and Janssen outside the submitted work. In addition, Dr. Rao is 492 co-inventor on a patent on Tph cells pending. Drs. Alves and Qu are employees of 493 Merck Sharp \& Dohme Corp., a subsidiary of Merck \& Co., Inc., Kenilworth, NJ, USA. 
494 Dr. Wang was an employee of Merck Sharp \& Dohme Corp., a subsidiary of Merck \&

495 Co., Inc., Kenilworth, NJ, USA during participation in this manuscript.

497 Reference

498 1. Choi MY, Barber MR, Barber CE, Clarke AE, Fritzler MJ. Preventing the

499 development of SLE: identifying risk factors and proposing pathways for clinical care.

500 Lupus. 2016 Jul;25(8):838-49. doi: 10.1177/0961203316640367. PMID: 27252260.

501 2. Amsden LB, Davidson PT, Fevrier HB, Goldfien R, Herrinton LJ. Improving

502 the quality of care and patient experience of care during the diagnosis of lupus: a

503 qualitative study of primary care. Lupus. 2018 Jun;27(7):1088-1099. doi:

504 10.1177/0961203318763082. Epub 2018 Mar 16. PMID: 29546773.

505 3. Kernder A, Richter JG, Fischer-Betz R, Winkler-Rohlfing B, Brinks R, Aringer

506 M, Schneider M, Chehab G. Delayed diagnosis adversely affects outcome in

507 systemic lupus erythematosus: Cross sectional analysis of the LuLa cohort. Lupus.

5082021 Mar;30(3):431-438. doi: 10.1177/0961203320983445. Epub 2021 Jan 5.

509 PMID: 33402036; PMCID: PMC7933718.

510 4. Mosca M, Costenbader KH, Johnson SR, Lorenzoni V, Sebastiani GD, Hoyer

511 BF, Navarra S, Bonfa E, Ramsey-Goldman R, Medina-Rosas J, Piga M, Tani C,

512 Tedeschi SK, Dörner T, Aringer M, Touma Z. Brief Report: How Do Patients With

513 Newly Diagnosed Systemic Lupus Erythematosus Present? A Multicenter Cohort of

514 Early Systemic Lupus Erythematosus to Inform the Development of New

515 Classification Criteria. Arthritis Rheumatol. 2019 Jan;71(1):91-98. doi:

516 10.1002/art.40674. Epub 2018 Nov 26. PMID: 30035365.

517 5. Arazi A, Rao DA, Berthier CC, Davidson A, Liu Y, Hoover PJ, Chicoine A,

518 Eisenhaure TM, Jonsson AH, Li S, Lieb DJ, Zhang F, Slowikowski K, Browne EP, 
519 Noma A, Sutherby D, Steelman S, Smilek DE, Tosta P, Apruzzese W, Massarotti E,

520 Dall'Era M, Park M, Kamen DL, Furie RA, Payan-Schober F, Pendergraft WF 3rd,

521 Mclnnis EA, Buyon JP, Petri MA, Putterman C, Kalunian KC, Woodle ES, Lederer

522 JA, Hildeman DA, Nusbaum C, Raychaudhuri S, Kretzler M, Anolik JH, Brenner MB,

523 Wofsy D, Hacohen N, Diamond B; Accelerating Medicines Partnership in SLE

524 network. The immune cell landscape in kidneys of patients with lupus nephritis. Nat

525 Immunol. 2019 Jul;20(7):902-914. doi: 10.1038/s41590-019-0398-x. Epub 2019 Jun

526 17. Erratum in: Nat Immunol. 2019 Aug 13;: PMID: 31209404; PMCID:

527 PMC6726437.

528 6. Der E, Suryawanshi H, Morozov P, Kustagi M, Goilav B, Ranabothu S, Izmirly 529 P, Clancy R, Belmont HM, Koenigsberg M, Mokrzycki M, Rominieki H, Graham JA,

530 Rocca JP, Bornkamp N, Jordan N, Schulte E, Wu M, Pullman J, Slowikowski K,

531 Raychaudhuri S, Guthridge J, James J, Buyon J, Tuschl T, Putterman C;

532 Accelerating Medicines Partnership Rheumatoid Arthritis and Systemic Lupus

533 Erythematosus (AMP RA/SLE) Consortium. Tubular cell and keratinocyte single-cell

534 transcriptomics applied to lupus nephritis reveal type I IFN and fibrosis relevant

535 pathways. Nat Immunol. 2019 Jul;20(7):915-927. doi: 10.1038/s41590-019-0386-1.

536 Epub 2019 May 20. Erratum in: Nat Immunol. 2019 Nov;20(11):1556. PMID:

537 31110316; PMCID: PMC6584054.

538 7. Banchereau R, Hong S, Cantarel B, Baldwin N, Baisch J, Edens M, Cepika

539 AM, Acs P, Turner J, Anguiano E, Vinod P, Kahn S, Obermoser G, Blankenship D,

540 Wakeland E, Nassi L, Gotte A, Punaro M, Liu YJ, Banchereau J, Rossello-Urgell J,

541 Wright T, Pascual V. Personalized Immunomonitoring Uncovers Molecular

542 Networks that Stratify Lupus Patients. Cell. 2016 Apr 21;165(3):551-65. doi: 
543 10.1016/j.cell.2016.03.008. Epub 2016 Mar 31. Erratum in: Cell. 2016 Jun

544 2;165(6):1548-1550. PMID: 27040498; PMCID: PMC5426482.

545 8. Hong S, Banchereau R, Maslow BL, Guerra MM, Cardenas J, Baisch J,

546 Branch DW, Porter TF, Sawitzke A, Laskin CA, Buyon JP, Merrill J, Sammaritano LR,

547 Petri M, Gatewood E, Cepika AM, Ohouo M, Obermoser G, Anguiano E, Kim TW,

548 Nulsen J, Nehar-Belaid D, Blankenship D, Turner J, Banchereau J, Salmon JE,

549 Pascual V. Longitudinal profiling of human blood transcriptome in healthy and lupus

550 pregnancy. J Exp Med. 2019 May 6;216(5):1154-1169. doi: 10.1084/jem.20190185.

551 Epub 2019 Apr 8. PMID: 30962246; PMCID: PMC6504211.

552 9. Amir el-AD, Davis KL, Tadmor MD, Simonds EF, Levine JH, Bendall SC,

553 Shenfeld DK, Krishnaswamy S, Nolan GP, Pe'er D. viSNE enables visualization of

554 high dimensional single-cell data and reveals phenotypic heterogeneity of leukemia.

555 Nat Biotechnol. 2013 Jun;31(6):545-52. doi: 10.1038/nbt.2594. Epub 2013 May 19.

556 PMID: 23685480; PMCID: PMC4076922.

557 10. Van Gassen S, Callebaut B, Van Helden MJ, Lambrecht BN, Demeester P,

558 Dhaene T, Saeys Y. FlowSOM: Using self-organizing maps for visualization and

559 interpretation of cytometry data. Cytometry A. 2015 Jul;87(7):636-45. doi:

560 10.1002/cyto.a.22625. Epub 2015 Jan 8. PMID: 25573116.

561 11. Bocharnikov AV, Keegan J, Wacleche VS, Cao Y, Fonseka CY, Wang G,

562 Muise ES, Zhang KX, Arazi A, Keras G, Li ZJ, Qu Y, Gurish MF, Petri M, Buyon JP,

563 Putterman C, Wofsy D, James JA, Guthridge JM, Diamond B, Anolik JH, Mackey MF,

564 Alves SE, Nigrovic PA, Costenbader KH, Brenner MB, Lederer JA, Rao DA;

565 Accelerating Medicines Partnership (AMP) RA/SLE Network. PD-1hiCXCR5- T

566 peripheral helper cells promote B cell responses in lupus via MAF and IL-21. JCI 
567 Insight. 2019 Oct 17;4(20):e130062. doi: 10.1172/jci.insight.130062. PMID:

568 31536480; PMCID: PMC6824311.

569 12. O'Gorman WE, Kong DS, Balboni IM, Rudra P, Bolen CR, Ghosh D, Davis

570 MM, Nolan GP, Hsieh EW. Mass cytometry identifies a distinct monocyte cytokine

571 signature shared by clinically heterogeneous pediatric SLE patients. J Autoimmun.

5722017 Apr 4:S0896-8411(16)30412-7. doi: 10.1016/j.jaut.2017.03.010. Epub ahead

573 of print. PMID: 28389038; PMCID: PMC5628110.

574 13. van der Kroef M, van den Hoogen LL, Mertens JS, Blokland SLM, Haskett S,

575 Devaprasad A, Carvalheiro T, Chouri E, Vazirpanah N, Cossu M, Wichers CGK,

576 Silva-Cardoso SC, Affandi AJ, Bekker CPJ, Lopes AP, Hillen MR, Bonte-Mineur F,

577 Kok MR, Beretta L, Rossato M, Mingueneau M, van Roon JAG, Radstake TRDJ.

578 Cytometry by time of flight identifies distinct signatures in patients with systemic

579 sclerosis, systemic lupus erythematosus and Sjögrens syndrome. Eur J Immunol.

5802020 Jan;50(1):119-129. doi: 10.1002/eji.201948129. Epub 2019 Sep 11. PMID:

58131424086.

582 14. Blanco P, Ueno H, Schmitt N. T follicular helper (Tfh) cells in lupus: Activation

583 and involvement in SLE pathogenesis. Eur J Immunol. 2016 Feb;46(2):281-90. doi:

584 10.1002/eji.201545760. PMID: 26614103.

585 15. van Vollenhoven RF, Hahn BH, Tsokos GC, Wagner CL, Lipsky P, Touma Z,

586 Werth VP, Gordon RM, Zhou B, Hsu B, Chevrier M, Triebel M, Jordan JL, Rose S.

587 Efficacy and safety of ustekinumab, an IL-12 and IL-23 inhibitor, in patients with

588 active systemic lupus erythematosus: results of a multicentre, double-blind, phase 2,

589 randomised, controlled study. Lancet. 2018 Oct 13;392(10155):1330-1339. doi:

590 10.1016/S0140-6736(18)32167-6. Epub 2018 Sep 21. PMID: 30249507. 
591 16. Arbuckle MR, McClain MT, Rubertone MV, Scofield RH, Dennis GJ, James

592 JA, Harley JB. Development of autoantibodies before the clinical onset of systemic

593 lupus erythematosus. N Engl J Med. 2003 Oct 16;349(16):1526-33. doi:

594 10.1056/NEJMoa021933. PMID: 14561795.

595 17. Rao DA, Gurish MF, Marshall JL, Slowikowski K, Fonseka CY, Liu Y, Donlin

596 LT, Henderson LA, Wei K, Mizoguchi F, Teslovich NC, Weinblatt ME, Massarotti EM,

597 Coblyn JS, Helfgott SM, Lee YC, Todd DJ, Bykerk VP, Goodman SM, Pernis AB,

598 Ivashkiv LB, Karlson EW, Nigrovic PA, Filer A, Buckley CD, Lederer JA,

599 Raychaudhuri S, Brenner MB. Pathologically expanded peripheral T helper cell

600 subset drives B cells in rheumatoid arthritis. Nature. 2017 Feb 1;542(7639):110-114.

601 doi: 10.1038/nature20810. PMID: 28150777; PMCID: PMC5349321.

602 18. Yoshitomi H, Ueno H. Shared and distinct roles of T peripheral helper and T

603 follicular helper cells in human diseases. Cell Mol Immunol. 2021 Mar;18(3):523-527.

604 doi: 10.1038/s41423-020-00529-z. Epub 2020 Aug 31. PMID: 32868910; PMCID:

605 PMC8027819.

606 19. Rao DA. T Cells That Help B Cells in Chronically Inflamed Tissues. Front

607 Immunol. 2018 Aug 23;9:1924. doi: 10.3389/fimmu.2018.01924. PMID: 30190721;

608 PMCID: PMC6115497.

609 20. Bashford-Rogers RJM, Bergamaschi L, McKinney EF, Pombal DC, Mescia F,

610 Lee JC, Thomas DC, Flint SM, Kellam P, Jayne DRW, Lyons PA, Smith KGC.

611 Analysis of the B cell receptor repertoire in six immune-mediated diseases. Nature.

6122019 Oct;574(7776):122-126. doi: 10.1038/s41586-019-1595-3. Epub 2019 Sep 25.

613 PMID: 31554970; PMCID: PMC6795535. 
614 21. Lycke NY, Bemark M. The regulation of gut mucosal IgA B-cell responses:

615 recent developments. Mucosal Immunol. 2017 Nov;10(6):1361-1374. doi:

616 10.1038/mi.2017.62. Epub 2017 Jul 26. PMID: 28745325.

617 22. Klemsz MJ, McKercher SR, Celada A, Van Beveren C, Maki RA. The

618 macrophage and B cell-specific transcription factor PU.1 is related to the ets

619 oncogene. Cell. 1990 Apr 6;61(1):113-24. doi: 10.1016/0092-8674(90)90219-5.

620 PMID: 2180582.

621 23. Wang R, Singaraju A, Marks KE, Shakib L, Dunlap G, Cunningham-Bussel A,

622 Chen L, Tirpack A, Fein MR, Todd DJ, MacFarlane L, Goodman SM, DiCarlo EF,

623 Massarotti EM, Sparks JA, Hamnvik OPR, Min L, Jonsson AH, Brenner MB, Chan

624 KK, Bass AR, Donlin LT, Rao DA. Clonally expanded CD38hi cytotoxic CD8 T cells

625 define the $\mathrm{T}$ cell infiltrate in checkpoint inhibitor-associated arthritis

626 24. Schmitt N, Liu Y, Bentebibel SE, Munagala I, Bourdery L, Venuprasad K,

627 Banchereau J, Ueno $\mathrm{H}$. The cytokine TGF- $\beta$ co-opts signaling via STAT3-STAT4 to

628 promote the differentiation of human TFH cells. Nat Immunol. 2014

629 Sep;15(9):856-65. doi: 10.1038/ni.2947. Epub 2014 Jul 27. PMID: 25064073;

630 PMCID: PMC4183221.

631 25. Locci M, Wu JE, Arumemi F, Mikulski Z, Dahlberg C, Miller AT, Crotty S.

632 Activin A programs the differentiation of human TFH cells. Nat Immunol. 2016

633 Aug;17(8):976-84. doi: 10.1038/ni.3494. Epub 2016 Jul 4. Erratum in: Nat Immunol.

6342016 Sep 20;17(10):1235. PMID: 27376469; PMCID: PMC4955732.

635 26. Morand EF, Furie R, Tanaka Y, Bruce IN, Askanase AD, Richez C, Bae SC,

636 Brohawn PZ, Pineda L, Berglind A, Tummala R; TULIP-2 Trial Investigators. Trial of

637 Anifrolumab in Active Systemic Lupus Erythematosus. N Engl J Med. 2020 Jan 
638 16;382(3):211-221. doi: 10.1056/NEJMoa1912196. Epub 2019 Dec 18. PMID:

63931851795.

640 27. Hochberg MC. Updating the American College of Rheumatology revised

641 criteria for the classification of systemic lupus erythematosus. Arthritis Rheum. 1997

642 Sep;40(9):1725. doi: 10.1002/art.1780400928. PMID: 9324032.

643 28. Finck R, Simonds EF, Jager A, Krishnaswamy S, Sachs K, Fantl W, Pe'er D,

644 Nolan GP, Bendall SC. Normalization of mass cytometry data with bead standards.

645 Cytometry A. 2013 May;83(5):483-94. doi: 10.1002/cyto.a.22271. Epub 2013 Mar 19.

646 PMID: 23512433; PMCID: PMC3688049.

647 29. Zunder ER, Finck R, Behbehani GK, Amir el-AD, Krishnaswamy S, Gonzalez

648 VD, Lorang CG, Bjornson Z, Spitzer MH, Bodenmiller B, Fantl WJ, Pe'er D, Nolan

649 GP. Palladium-based mass tag cell barcoding with a doublet-filtering scheme and

650 single-cell deconvolution algorithm. Nat Protoc. 2015 Feb;10(2):316-33. doi:

651 10.1038/nprot.2015.020. Epub 2015 Jan 22. PMID: 25612231; PMCID:

652 PMC4347881.

\section{Figure legends}

655 Figure 1. Overview of immunophenotyping early SLE using cross-sectional

656 and longitudinal samples. (A) PBMCs were acquired from 14 controls, nine early

657 SLE, and 15 established SLE and profiled with three different mass cytometry

658 panels. (B) For the nine early SLE patients, longitudinal PBMC and serum samples

659 were collected reflecting three timepoints: at enrollment (time A), six months after

660 enrollment (time B), and 12 months after enrollment (time C). Immune cell frequency

661 was assessed with mass cytometry and serum cytokines were assessed by Luminex

662 cytokines assay. (C) Immunophenotyping panel mass cytometry data from nine 
663 early SLE and 15 established SLE were merged and visualized with tSNE plots.

664 Frequencies of labelled immune cell types were compared between controls, early

665 SLE, and established SLE cohorts by Kruskal-Wallis with Dunn's test of multiple comparisons. ${ }^{\star} \mathrm{P}<0.05,{ }^{* *} \mathrm{P}<0.01,{ }^{* * *} \mathrm{P}<0.001$. Data are shown as mean $\pm \mathrm{SE}$.

Figure 2. Expanded $\mathrm{Ki}^{+} \mathrm{ICOS}^{+} \mathrm{CD} 8 \mathrm{~T}$ cells in early SLE. (A) Abundance (\% of 669 total CD8 T cells) of FlowSOM metaclusters of CD8 ${ }^{+}$T cells in 14 controls, nine early 670 SLE and 15 established SLE. Kruskal-Wallis with Dunn's test was used for 671 comparisons. (B) Heatmap of normalized expression of mass cytometry markers in 672 each metacluster. Markers with average of metacluster medians $>0.2$ are shown.

673 (C) tSNE visualization of CD8 T cells from nine early SLE patients. The orange 674 circle: $\mathrm{Ki}_{67}^{+} \mathrm{ICOS}^{+}$CD8 cells. (D) Representative gating for $\mathrm{Ki} 7^{+} \mathrm{ICOS}^{+} \mathrm{CD} 8 \mathrm{~T}$ cell 675 population in $\mathrm{CD}^{+} \mathrm{CD}^{-} \mathrm{CD}^{-} \mathrm{CD}^{+}$cells, and the comparison between 14 controls, 676 nine early SLE, and 15 established SLE. (E) Longitudinal changes of $\mathrm{Ki}^{+} 7^{+} \mathrm{ICOS}^{+}$

677 CD8 T cell frequency in early SLE at enrollment (time A), six months after enrollment 678 (time B), and 12 months after enrollment (time C). Wilcoxon log-rank test to compare 679 between $A$ and $C$ was used for $p$-value calculation. (F) Longitudinal changes of 680 disease activity by SLEDAI in early SLE patients as in $(E) .{ }^{*} P<0.05,{ }^{* *} P<0.01$, $681{ }^{* * *} \mathrm{P}<0.001$. Data are shown as mean $\pm \mathrm{SE}$.

683 Figure 3. Distinct longitudinal changes of Tph cells and Tfh cells in early SLE.

684 (A) Abundance (\% of total CD4 T cells) of FlowSOM metaclusters of CD4 ${ }^{+}$T cells in 68514 controls, nine early SLE and 15 established SLE. Kruskal-Wallis with Dunn's 686 multiple test was used for comparisons. (B) Heatmap of normalized expression of 687 mass cytometry markers in each metacluster. Markers with average of metacluster 
688 medians > 0.2 are shown. (C) tSNE visualization of CD4 T cells in nine early SLE

689 patients. The orange circle: Tfh cells, the green circle: Tph cells, the grey circle:

690 Ki67 ${ }^{\text {hi }}$ cells Treg cells. (D, E) Representative gating for Tph cells, Tfh cells, and Ki67 ${ }^{\text {hi }}$

691 Treg in $\mathrm{CD}^{+} \mathrm{CD}^{-} \mathrm{CD}^{+} \mathrm{CD}^{-} \mathrm{CD} 45 \mathrm{RO}^{+}$memory CD4 T cells, and the comparison

692 between 14 controls, nine early SLE, and 15 established SLE. Kruskal-Wallis with

693 Dunn's multiple test was used for comparisons. (F) Longitudinal changes of Tph

694 cells, Tfh cells, and Ki67 ${ }^{+}$Treg cell in early SLE at enrollment (time A), six months

695 after enrollment (time B), and 12 months after enrollment (time C). Wilcoxon log-rank

696 test to compare between $A$ and $C$ was used for calculation of p-value. (G)

697 Correlation between prednisone dose and Tfh cell frequency in 27 datapoints (nine

698 early SLE patients $x$ three timepoints each). Spearman statistics shown. $(\mathrm{H}) \mathrm{Tfh}$

699 frequency in early SLE patients with or without MMF treatment as in $(G)(n=27$ data

700 points). Mann-Whitney $U$ test was used for the comparison. ${ }^{*} \mathrm{P}<0.05,{ }^{*} \mathrm{P}<0.01$,

$701 \quad{ }^{* *} \mathrm{P}<0.001$. Data are shown as mean $\pm \mathrm{SE}$.

702

703 Figure 4. Expanded ABCs and plasmablasts in early SLE patients.

704 (A) Abundance (\% of total B cells) of FlowSOM metaclusters of B cells in 14 controls,

705 nine early SLE and 15 established SLE. Kruskal-Wallis with Dunn's multiple test was

706 used for comparisons. (B) Heatmap of normalized expression of mass cytometry

707 markers in each metacluster. Markers with average of metacluster medians $>0.2$

708 are shown. (C) tSNE visualization of CD19+ B cells in nine early SLE patients. The

709 orange circle: CD19 ${ }^{\text {int }} \mathrm{Ki67} 7^{\text {hi }}$ plasmablasts, the green circle: ABCs. (D-F)

710 Representative gating for CD19 ${ }^{\text {int }} \mathrm{Ki} 67^{\text {hi }}$ plasmablasts, $\mathrm{ABC}$, and $\operatorname{lgM}^{+} \operatorname{lgD}^{+} \mathrm{ABCs}$ in

$711 \mathrm{CD}^{\mathrm{C}} \mathrm{CD}^{+} \mathrm{C}^{-} \mathrm{B}$ cells, and the comparison between 14 controls, nine early SLE, and

71215 established SLE. Kruskal-Wallis with Dunn's multiple test was used for 
713 comparisons. (G) Proportion of $\operatorname{lgG}^{+}$and $\lg \mathrm{A}^{+}$cells in $\mathrm{CD}^{\mathrm{int}} \mathrm{Ki}^{\mathrm{i}} 7^{\mathrm{hi}}$ plasmablasts

714 and ABCs in nine early SLE and 15 established SLE patients. Wilcoxon

715 matched-pair signed rank test was used for the comparison. $(\mathrm{H})$ Longitudinal

716 changes of $\operatorname{lgG}^{+} \mathrm{CD}_{19}{ }^{\text {int }} \mathrm{Ki} 7^{\text {hi }}$ plasmablasts, $\lg \mathrm{A}^{+} \mathrm{CD} 19^{\text {int }} \mathrm{Ki} 7^{\text {hi }}$ plasmablasts, $\operatorname{lgG}^{+}$

$717 \mathrm{ABCs}$, and $\lg \mathrm{A}^{+} \mathrm{ABCs}$ in early SLE at enrollment (time $\mathrm{A}$ ), six months after

718 enrollment (time B), and 12 months after enrollment (time C). Wilcoxon matched-pair

719 signed rank test to compare between $A$ and $C$ was used for calculation of $P$ value.

$720{ }^{*} \mathrm{P}<0.05,{ }^{* \star} \mathrm{P}<0.01,{ }^{* * *} \mathrm{P}<0.001$. Data are shown as mean $\pm \mathrm{SE}$.

722 Figure 5. PU.1 ${ }^{\text {hi }}$ Ki67 $^{\text {hi }}$ monocytes were expanded in early SLE.

723 (A) Abundance (\% of total monocytes) of FlowSOM metaclusters of monocytes in 14

724 controls, nine early SLE and 15 established SLE. Kruskal-Wallis with Dunn's

725 multiple test was used for comparisons. Kruskal-Wallis with Dunn's multiple test was

726 used for comparisons. (B) Heatmap of normalized expression of mass cytometry

727 markers in each metacluster. Expression of PU.1, CD69, Ki67, CCR2, CD14,

728 HLA-DR, CD16 are shown. (C) tSNE visualization of CD14 monocytes in nine early

729 SLE. The orange circle: PU. $1^{\text {hi }}$ Ki67 $7^{\text {hi }}$ monocytes. (D) correlation of metacluster 13

730 with HLA-DR Ki67 $^{\text {hi }}$ monocytes and PU. $1^{\text {hi }}$ Ki67 $7^{\text {hi }}$ monocytes. Spearman correlation

731 statistics shown. Solid line represents line of best fit. (E) Representative gating for

732 PU. $1^{\text {hi }} \mathrm{Ki} 67^{\text {hi }}$ monocytes and the comparison between 14 controls, nine early SLE,

733 and 15 established SLE. Kruskal-Wallis with Dunn's multiple test was used for

734 comparisons. (F) CCR2 expression on PU. $1^{\text {hi }} \mathrm{Ki} 67^{\text {hi }}$ monocytes and PU. $1^{\text {low }}$ Ki67 ${ }^{\text {low }}$

735 monocytes (nine early SLE and 15 established SLE). (G) Longitudinal changes of

736 PU. $1^{\text {hi }} \mathrm{Ki}^{\mathrm{hi}}$ monocytes in 9 early SLE at enrollment (time A), six months after

737 enrollment (time B), and 12 months after enrollment (time C). Wilcoxon log-rank test 
738 to compare between $A$ and $C$ was used for calculation of $P$ value. ${ }^{*} P<0.05,{ }^{* \star} P<0.01$, $739{ }^{* *} \mathrm{P}<0.001$. Data are shown as mean $\pm \mathrm{SE}$.

741 Figure 6. Association of Tph, Tfh, and Ki67 proliferative immune cells in early 742 SLE.

743 (A) Representative gating for NKG2D+ $\mathrm{Ki}^{+} 7^{+} \mathrm{NK}$ cells and the comparison between 74414 controls, nine early SLE, and 15 established SLE. Kruskal-Wallis with Dunn's 745 multiple test was used for comparisons. (B) Longitudinal changes of NKG2D+ $\mathrm{Ki}^{+} 7^{+}$

746 NK cells in nine early SLE patients at enrollment (time A), six months after

747 enrollment (time B), and 12 months after enrollment (time C). Wilcoxon log-rank test

748 to compare between $A$ and $C$ was used for calculation of $P$ value. $(C)$ tSNE

749 visualization of NKG2D+ $\mathrm{Ki}^{+} 7^{+} \mathrm{NK}$ cells in 14 controls, nine early SLE, and 15

750 established SLE. The orange circle: NKG2D+ Ki67 ${ }^{+}$NK cells. (D) Hierarchical

751 clustering heatmap with expanded immune cell types in early SLE. 27 data points

752 from early SLE patients (nine patients $x$ three time points) were used for this analysis.

753 Correlation coefficients calculated by Spearman's test were used for the heatmap.

754 (E) Correlation analysis between Tph cells, Tfh cells, ABCs, and CD19 ${ }^{\text {int }} \mathrm{Ki}^{\mathrm{int}}$

755 plasmablasts. 27 early SLE patient data points were used as in (D). Spearman

756 correlation statistics shown. Solid line represents line of best fit. ${ }^{*} P<0.05,{ }^{* *} P<0.01$,

$757{ }^{* *} \mathrm{P}<0.001$. Data are shown as mean $\pm \mathrm{SE}$.

759 Figure 7. Dysregulated cytokine- and chemokine-networks in early SLE.

760 (A) A hierarchical clustering heatmap with serum cytokines and chemokines in early

761 SLE patients. 65 cytokines were assessed by Luminex assay, and 33 cytokines

762 were detected. 27 data points from early SLE patients (nine patients $x$ three time 
763 points) were used for this analysis. Correlation coefficients calculated by

764 Spearman's test were used for the heatmap. (B) Comparison of serum cytokine

765 levels between nine controls and nine early SLE (time A). 15 cytokines were

766 significantly different between controls and early SLE (time A). Mann-Whitney U test

767 was used for the comparison. (C) Longitudinal changes of CXCL10, CD40L, IL-20,

768 and TWEAK in nine early SLE at enrollment (time A), six months after enrollment

769 (time B), and 12 months after enrollment (time C). Wilcoxon log-rank test to compare

770 between $A$ and $C$ was used for calculation of $p$ value. (D) A hierarchical clustering

771 heatmap with expanded immune cell types and chemokines in early SLE. 27 data

772 points from early SLE patients (nine patients $x$ three time points) were used for this

773 analysis. Correlation coefficients calculated by Spearman's test were used for the

774 heatmap. ${ }^{*} \mathrm{P}<0.05,{ }^{* *} \mathrm{P}<0.01,{ }^{* * *} \mathrm{P}<0.001$. Data are shown as mean $\pm \mathrm{SE}$.

775

776 Figure 8. Dysregulated immune cell types, cytokines, chemokines, and

777 chronological changes in early SLE. The increase pattern of cell types and

778 cytokines/chemokines levels at the diagnosis and one year after the diagnosis in

779 early SLE.

780

781

782

783

784

785

786 
bioRxiv preprint doi: https://doi.org/10.1101/2021.11.08.467791; this version posted November 10,2021 . The copyright holder for this preprint (which was not certified by peer review) is the author/funder, who has granted bioRxiv a license to display the preprint in perpetuity. It is made available under aCC-BY-NC-ND 4.0 International license.

788

789

790

791

792

793

794 
bioRxiv preprint doi: https:/doi.org/10.1101/2021.11.08.467791: this version posted November 10,2021 . The copyright holder for this preprint (which was not certified by peer review) is the author/funder, who has granted bioRxiv a license to display the preprint in perpetuity. It is made

Figure 1

A

Cross-sectional cohort

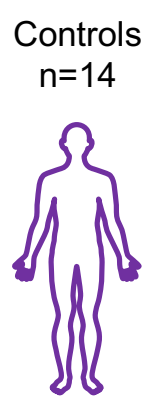

Established SLE

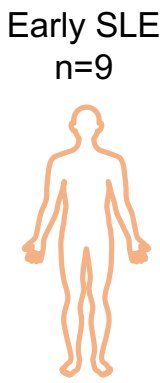

PBMCs

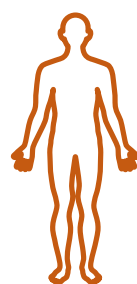

Longitudinal cohort

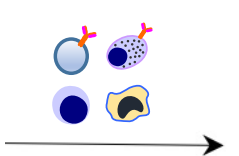

Mass cytometry
Metaclustering comparison (FlowSOM)

B

Timeline

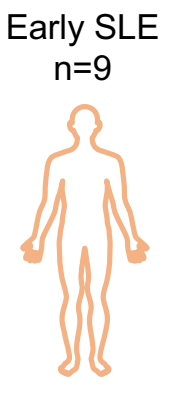

Time C

Time B 12 months

Time A 6 months

Enrollment
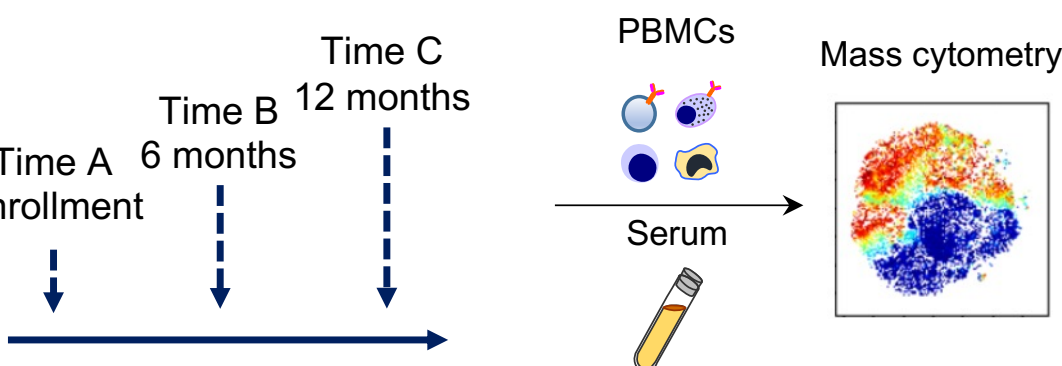

Luminex Assay 65 cytokines

Immunophenotyping panel $=39$ markers

$\mathrm{T}$ cell $/$ monocyte panel $=39$ markers

$\mathrm{B}$ cell panel $=38$ markers

C

CD3

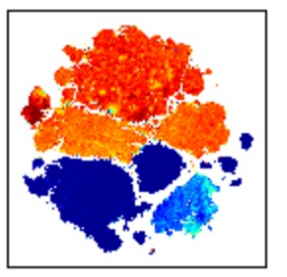

CD56

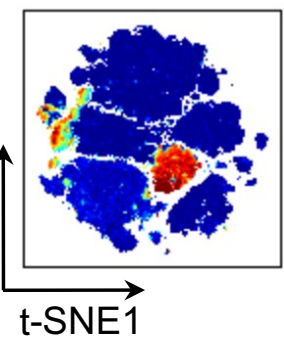

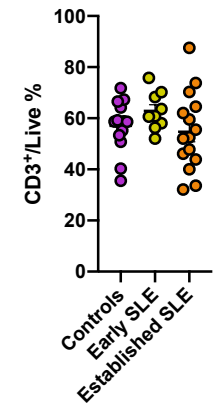

T cells

NK

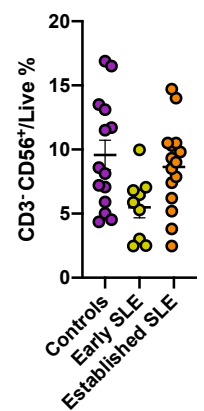

CD8 T

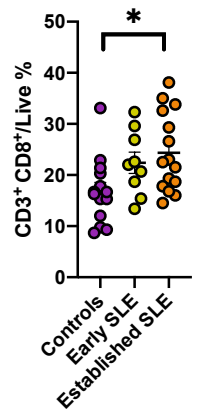

B cells

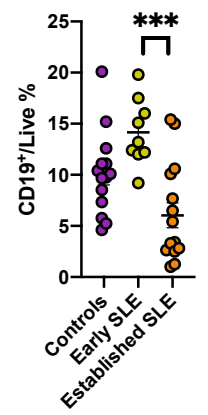

CD19

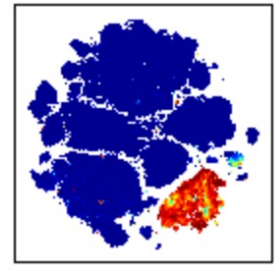

CD4 T

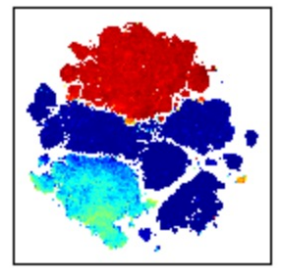

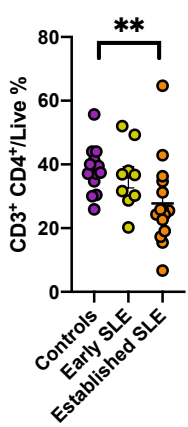

Monocytes

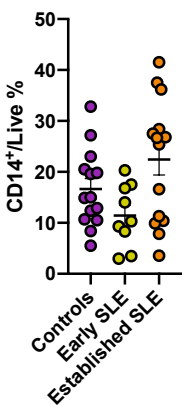

$\mathrm{TCR} \gamma \delta$

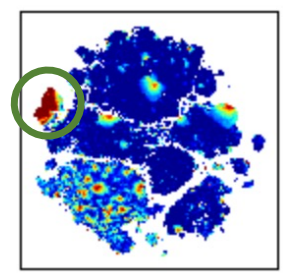

CD11c
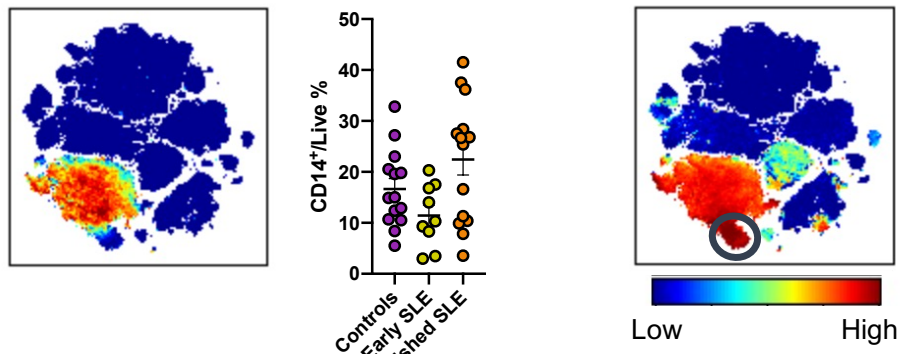

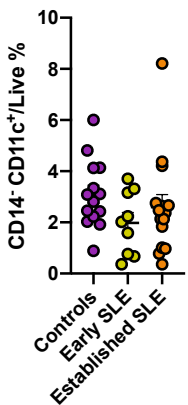


A
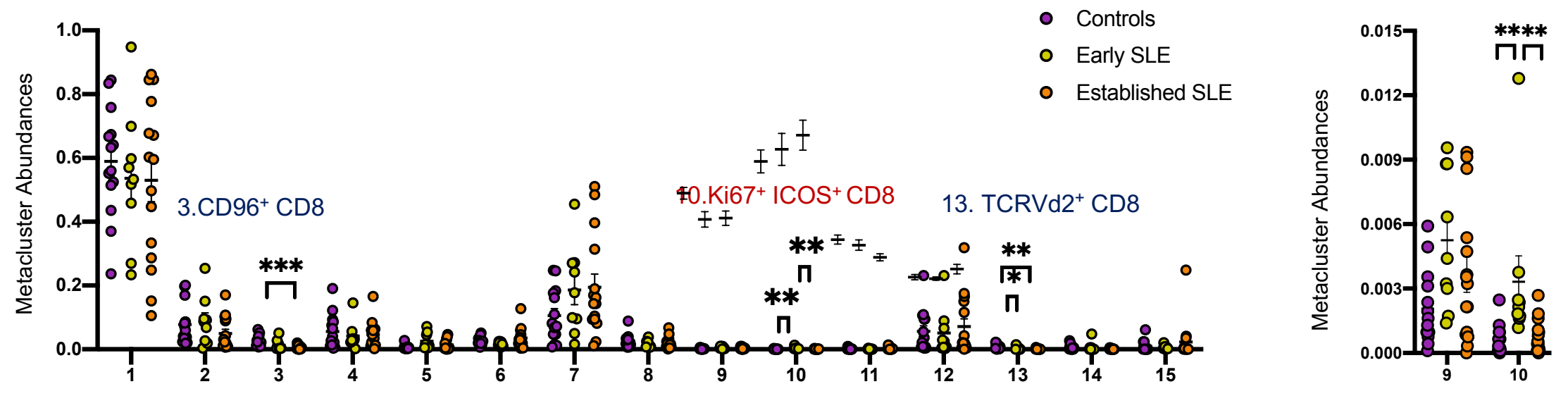

B

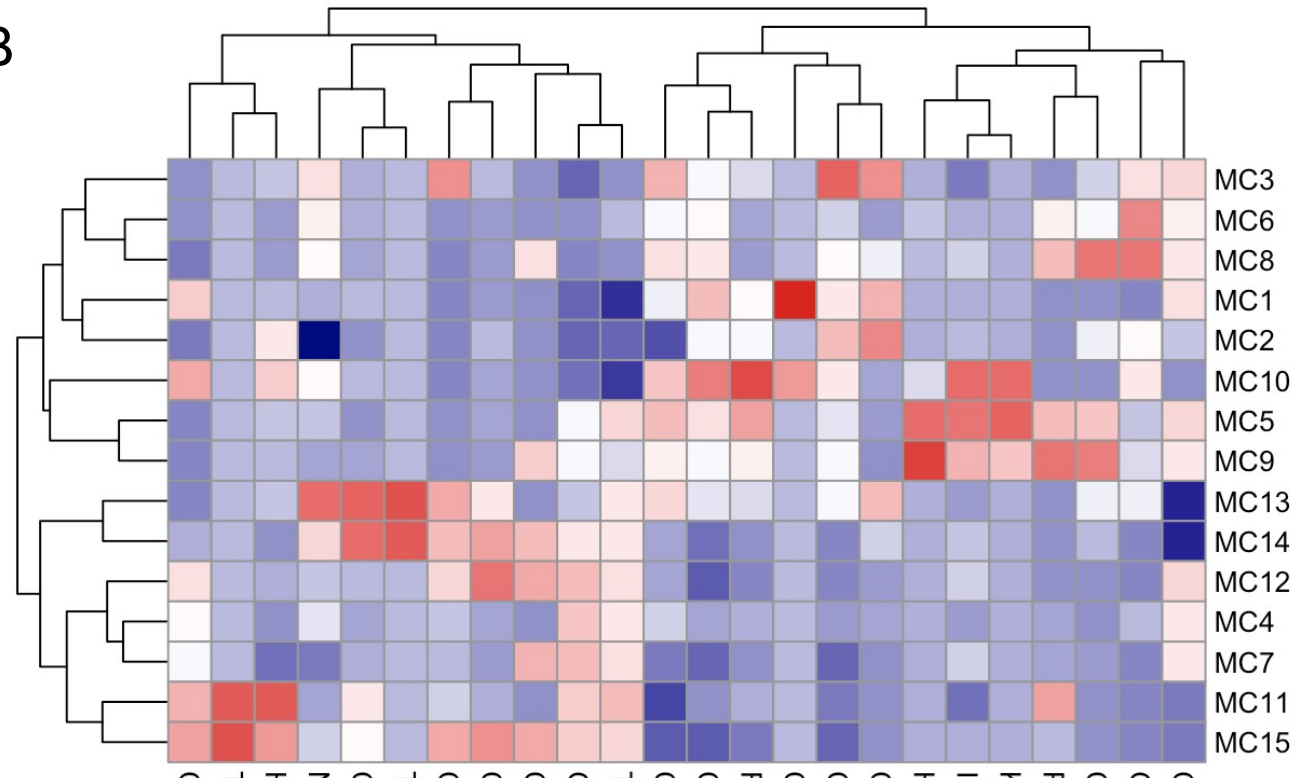

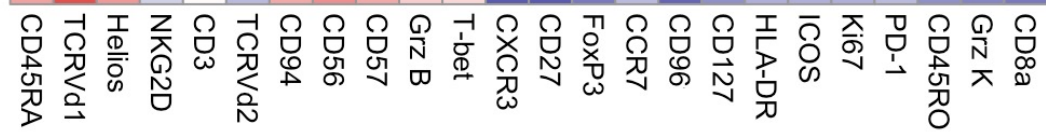

Z-score

$\begin{array}{ll}-2 & 2\end{array}$

C

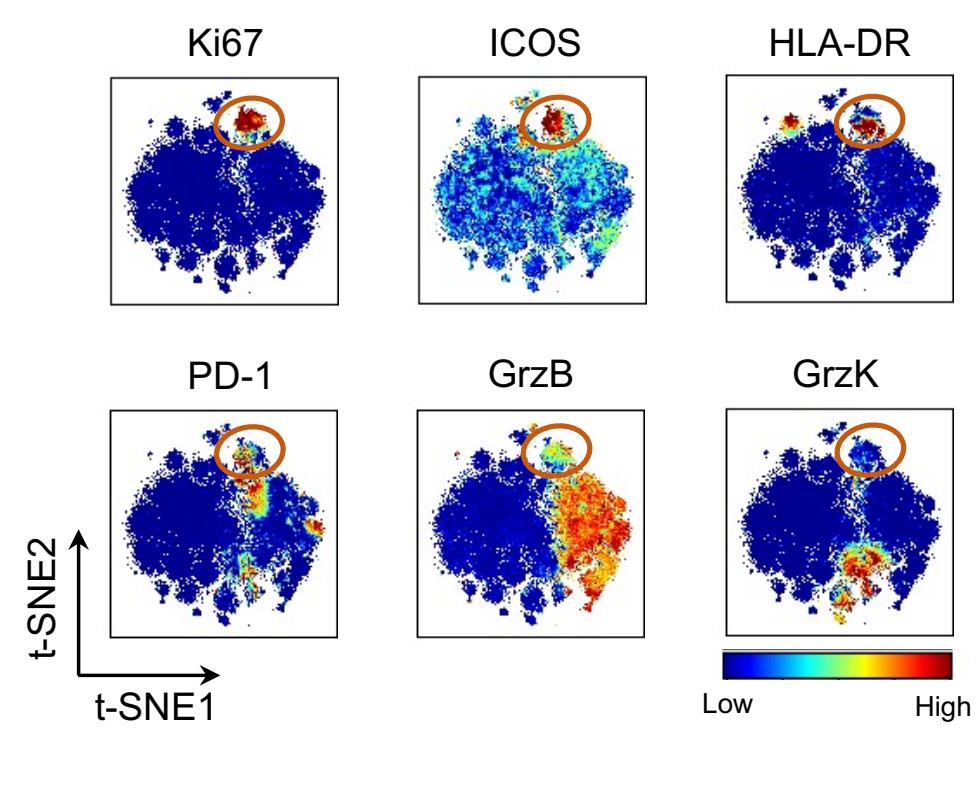

D

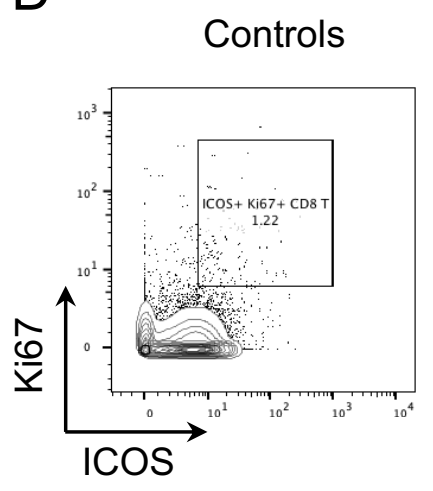

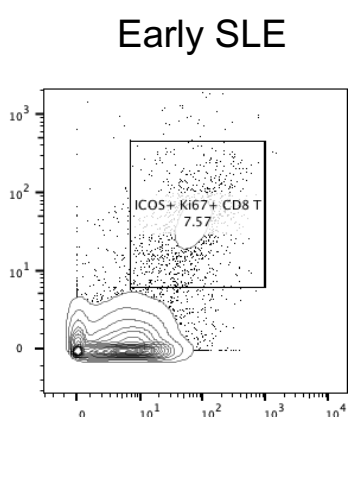

E

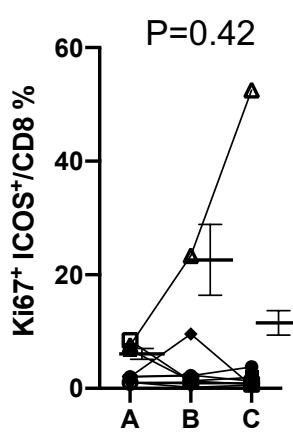

F

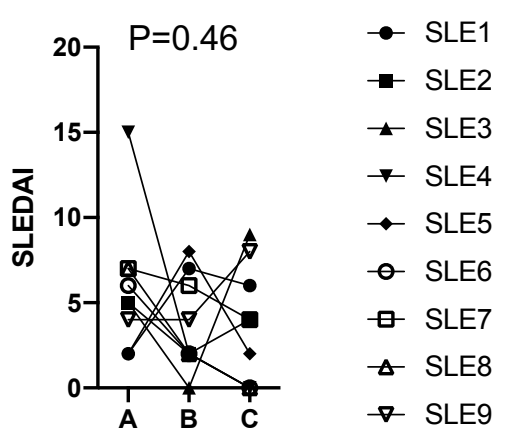


A

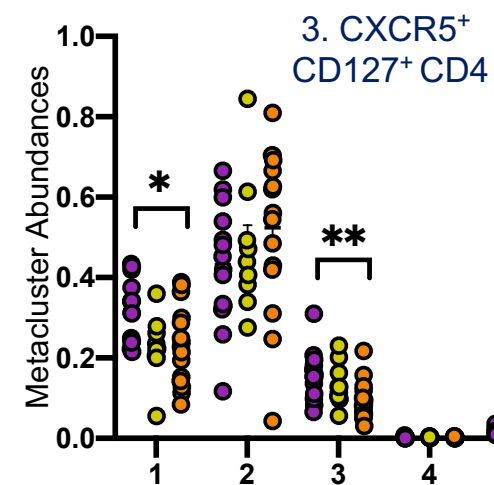

7. Cytotoxic CD4

華 $* * * * *$

1. CXCR5

CD $127^{+}$CD4

B

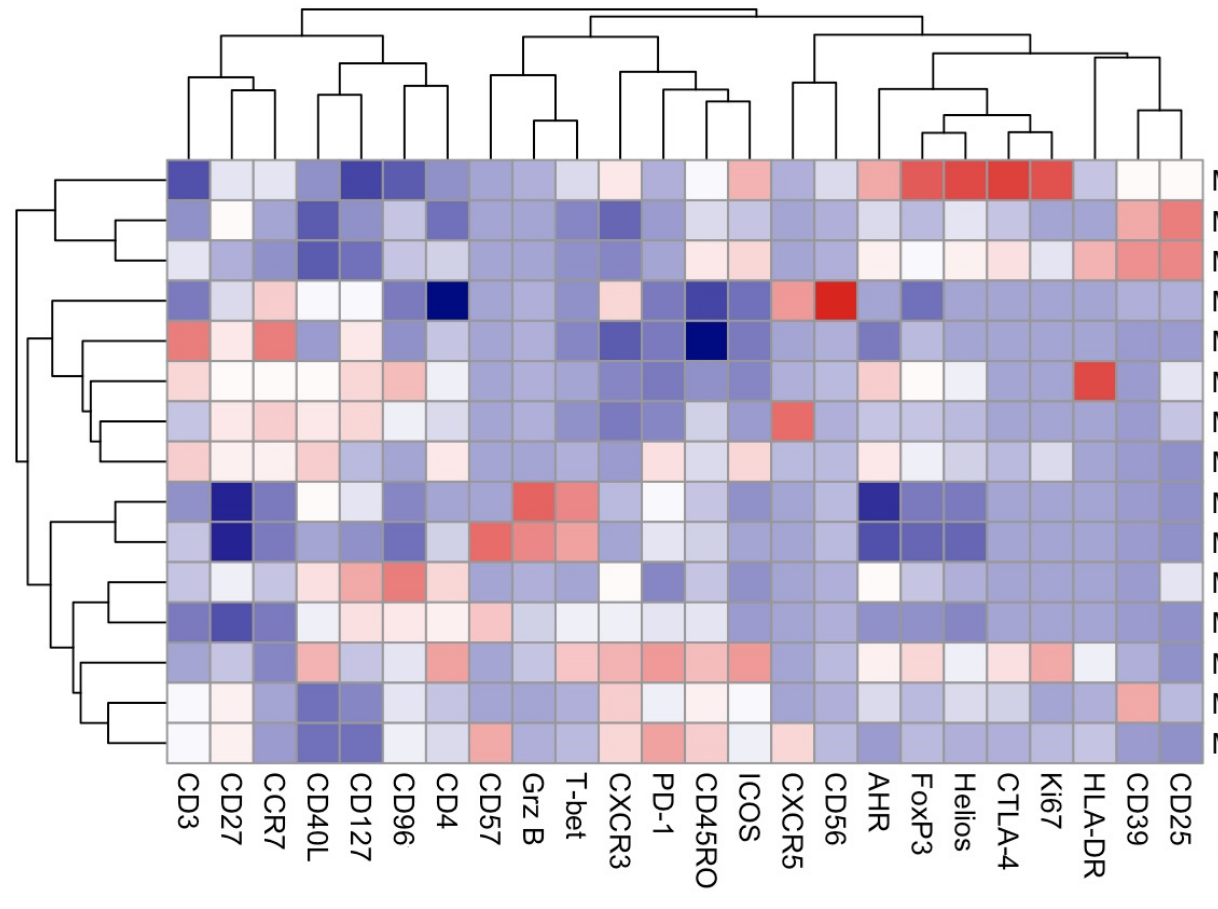

- Controls

- Early SLE

- Established SLE

13. $\mathrm{CXCR}^{+} \mathrm{Tph}$

15. Ki67 ${ }^{+}$Treg

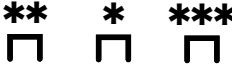

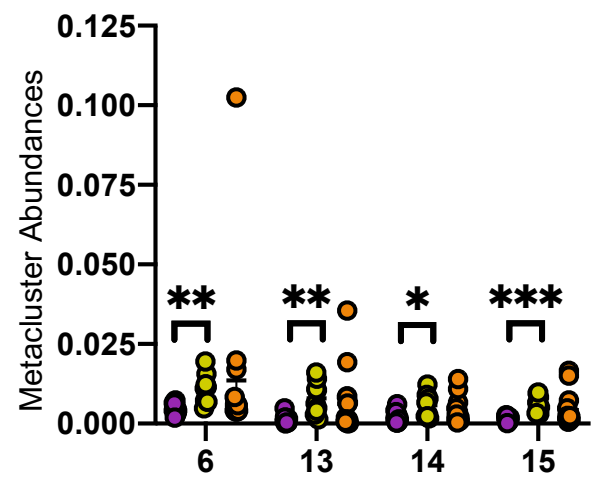

14. Tfh

C

D Controls

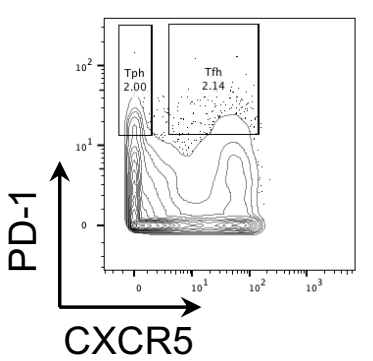

F

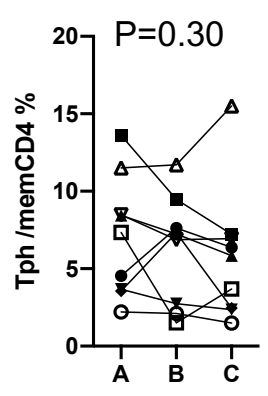

Early SLE
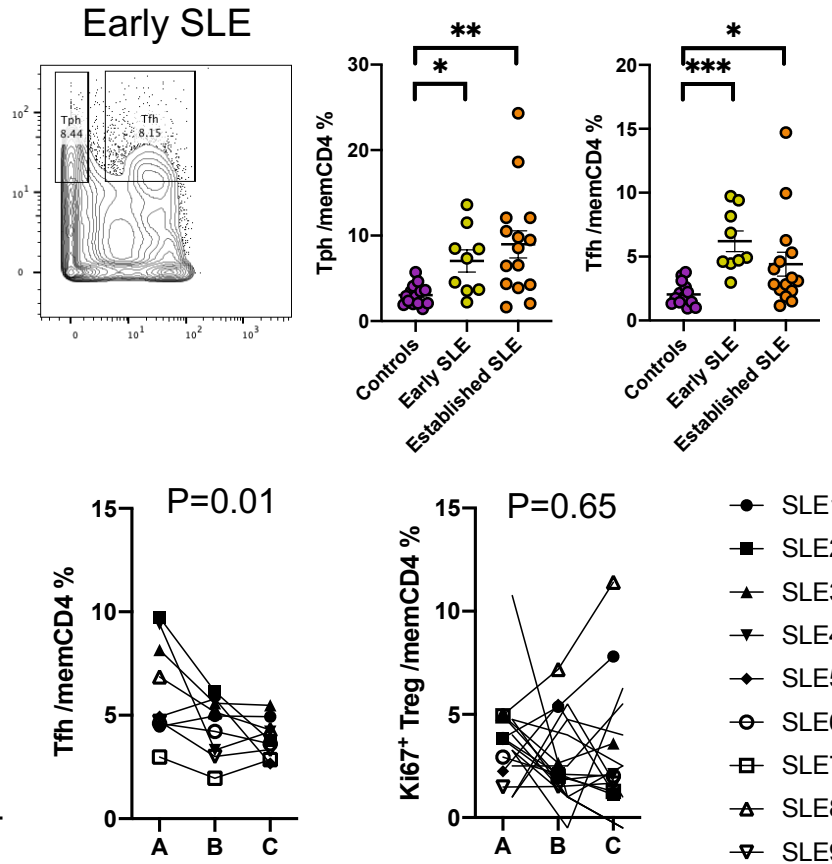

MC15
MC10
MC11
MC4
MC2
MC12
MC3
MC6
MC7
MC8
MC1
MC9
MC13
MC5
MC14

\section{Z-score}
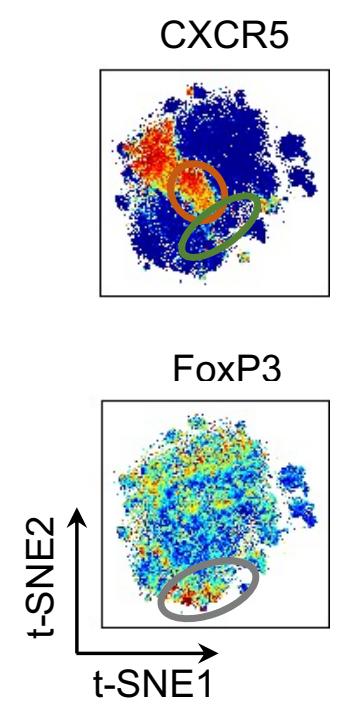

$-2 \quad 2$
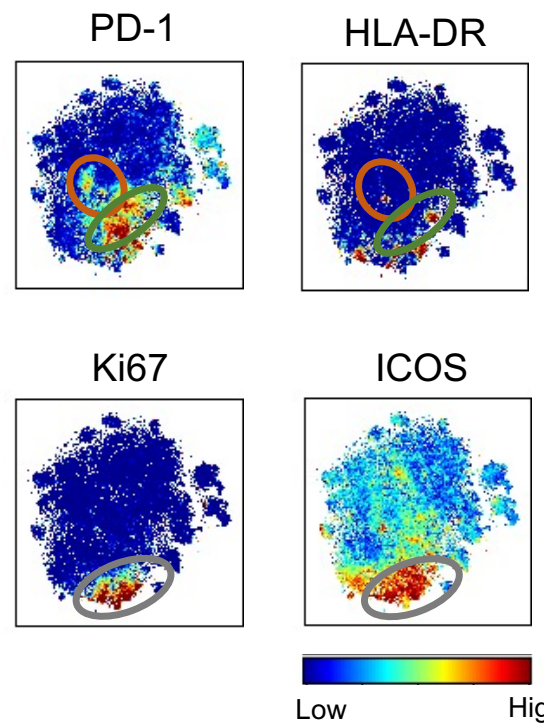

E

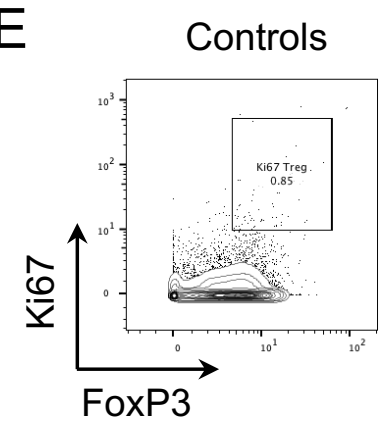

$G$

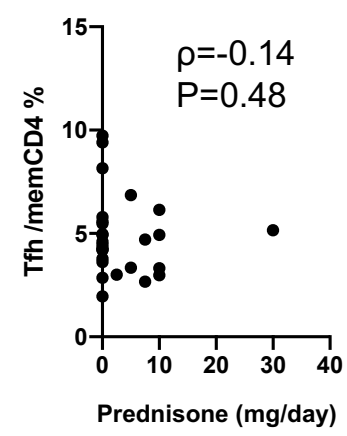

Early SLE

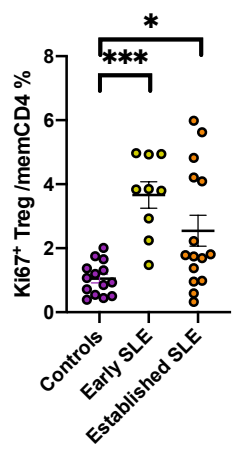

$\mathrm{H}$

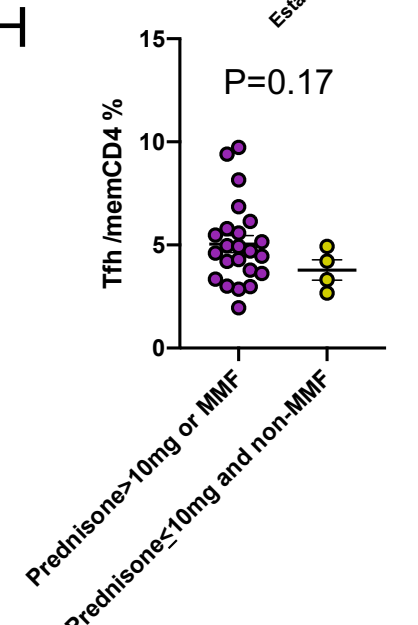


A

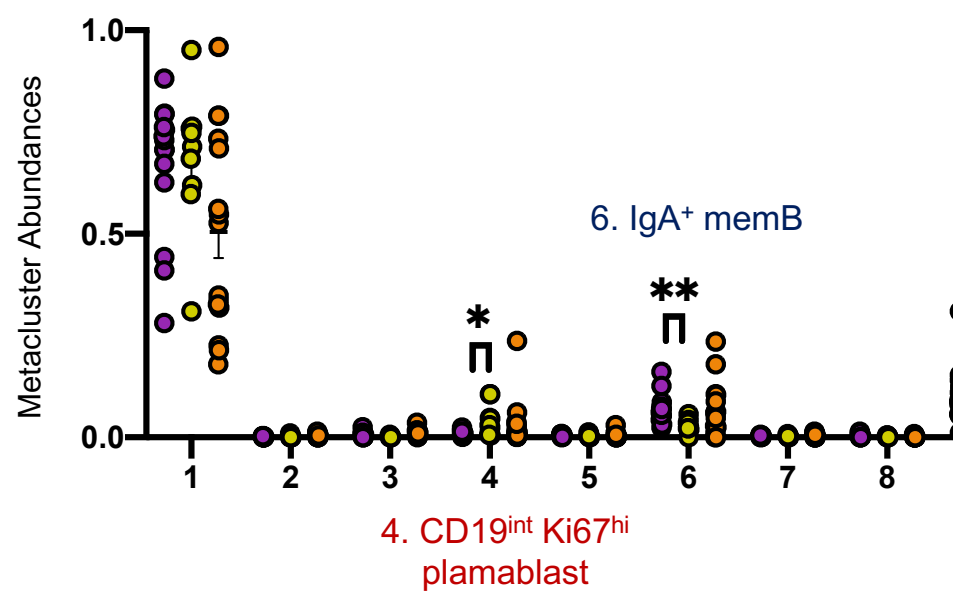

B

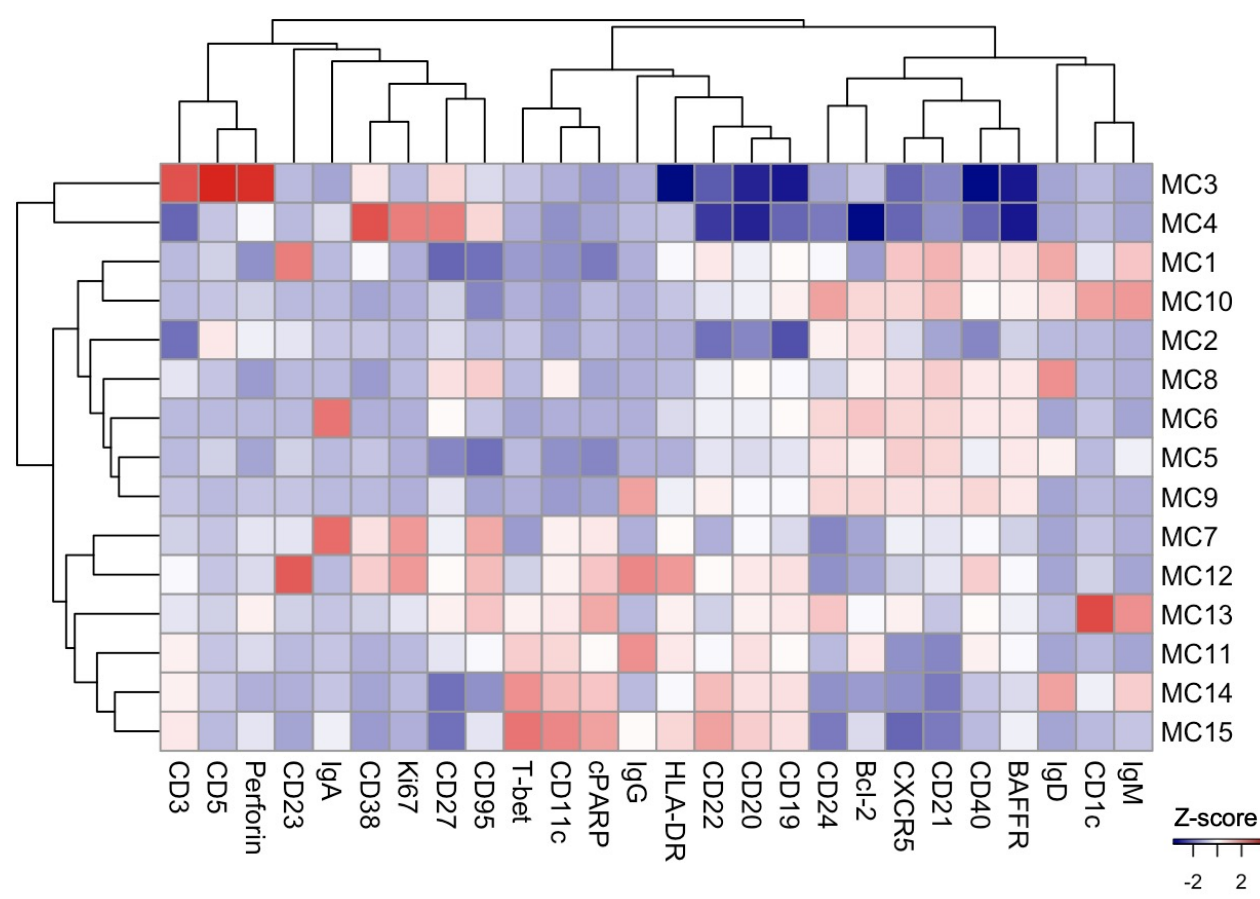

- Controls

- Early SLE

- Established SLE

15. CD11chi Tbethi ABCs

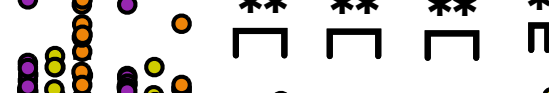

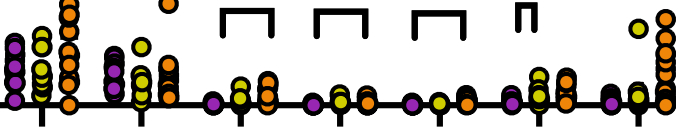

11,12.lgG ${ }^{+} A B C s$ 14. $\lg M^{+} \lg D^{+} A B C s$

\section{3. $\mathrm{CD}_{1 \mathrm{C}^{+}} \operatorname{lgM}^{+} \mathrm{ABCs}$}

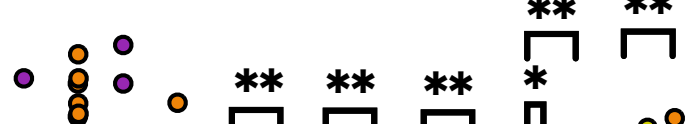

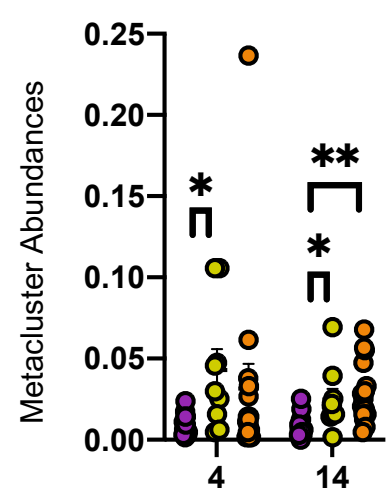

C
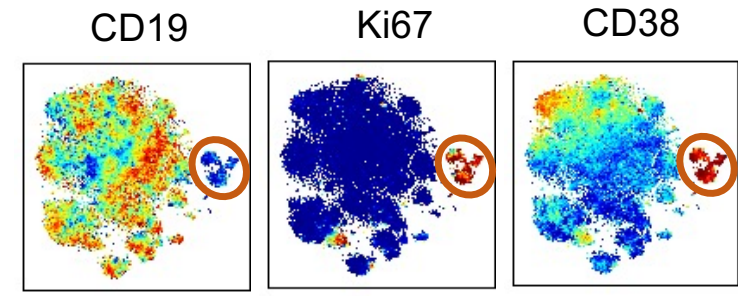

BAFF-R
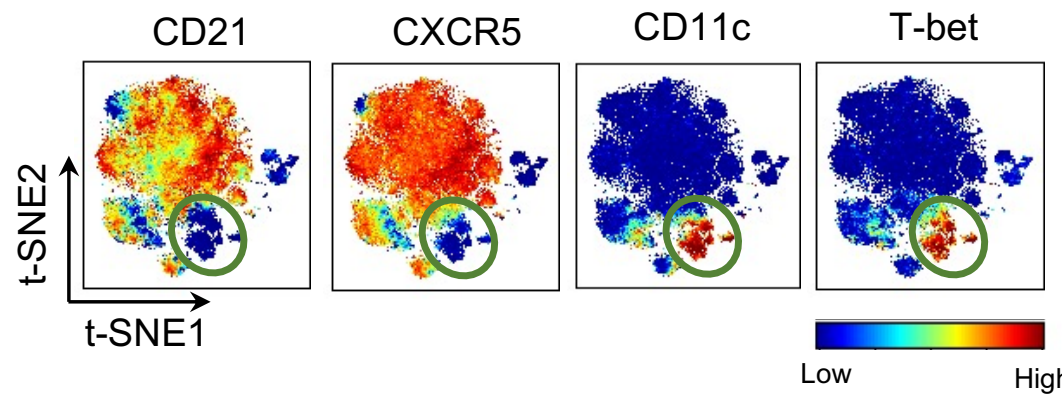

E
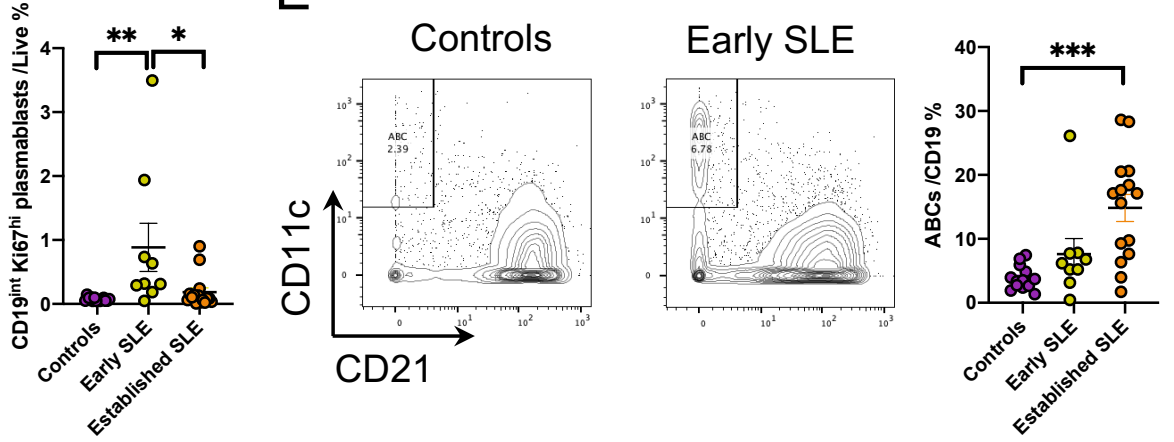

$F$

Controls $\quad * \quad \mathrm{G}$
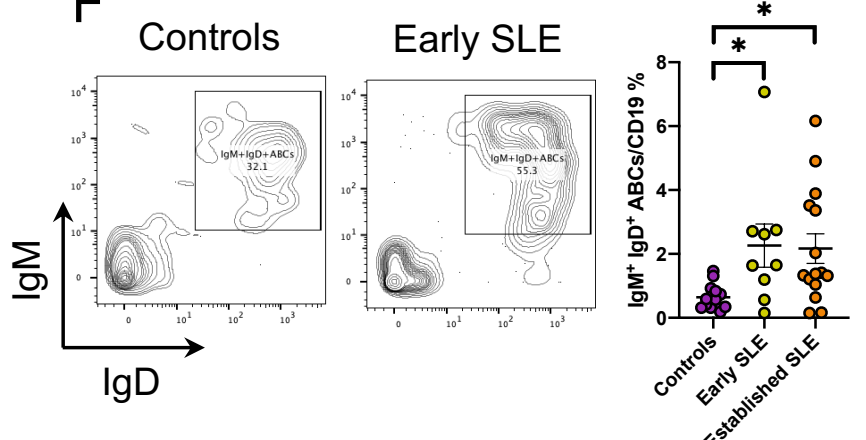

$\mathrm{H}$

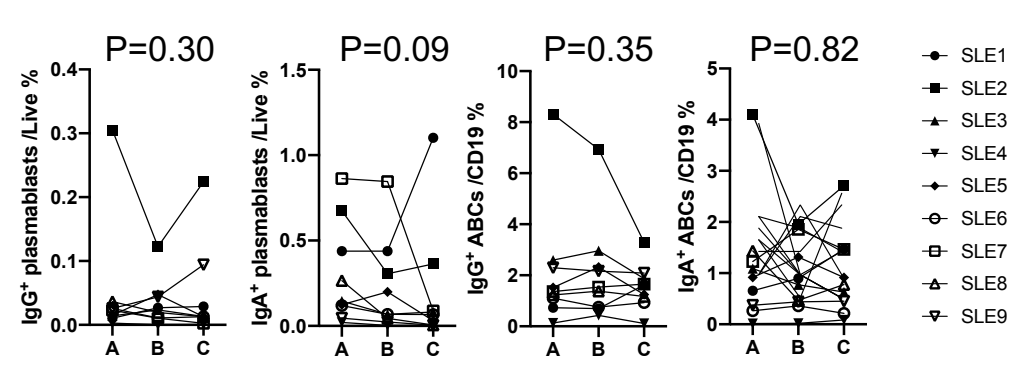


bioRxiv preprint doi: https://doi.org/10.1101/2021.11.08 467791: this version posted November 10,2021 . The copyright holder for this preprint Figu(wajeh not certified by peer review) is the author/funder, who has granted bioRxiv a license to display the preprint in perpetuity. It is made

A

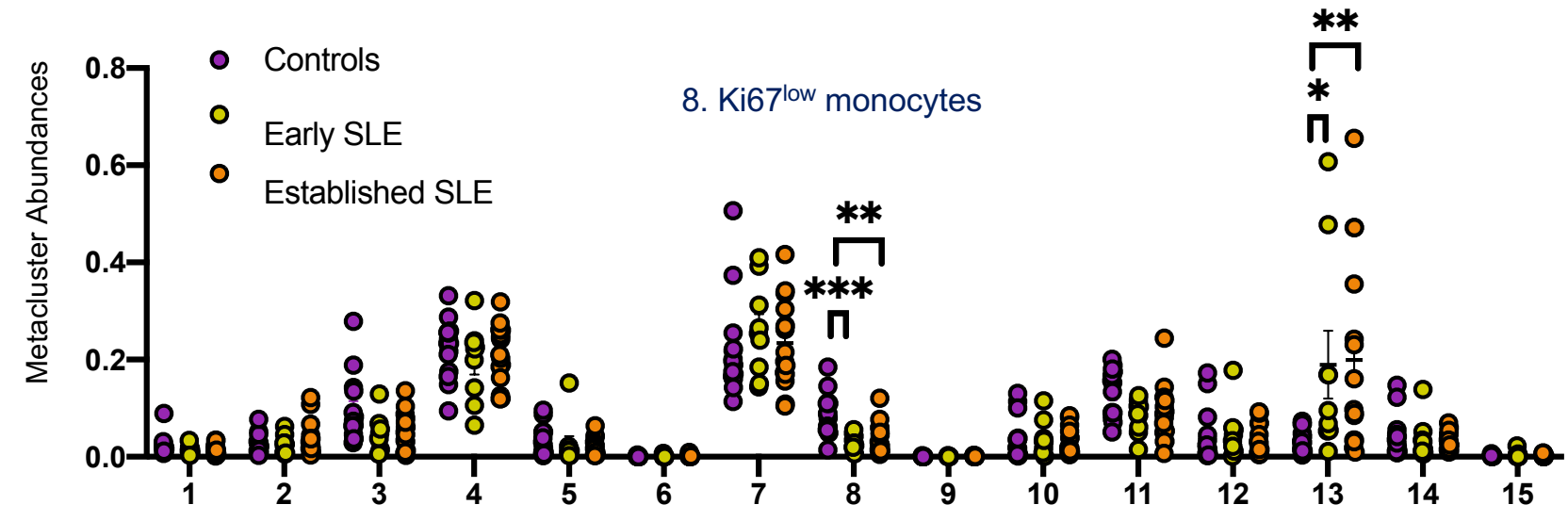

C
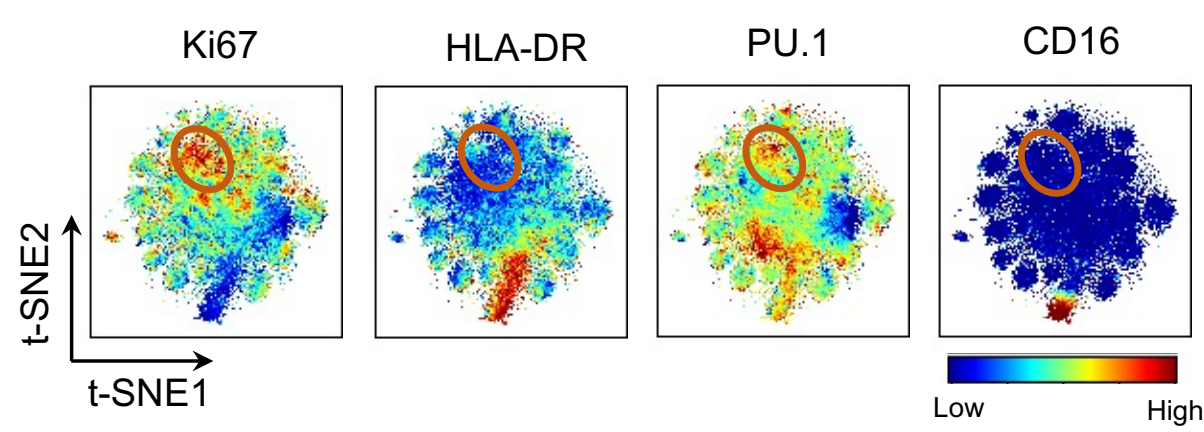

D

E
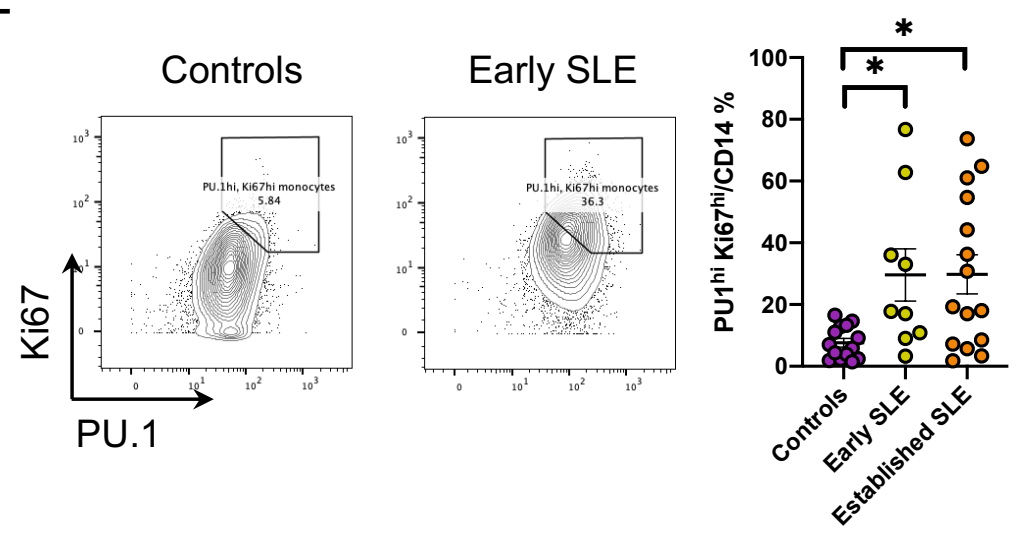

F

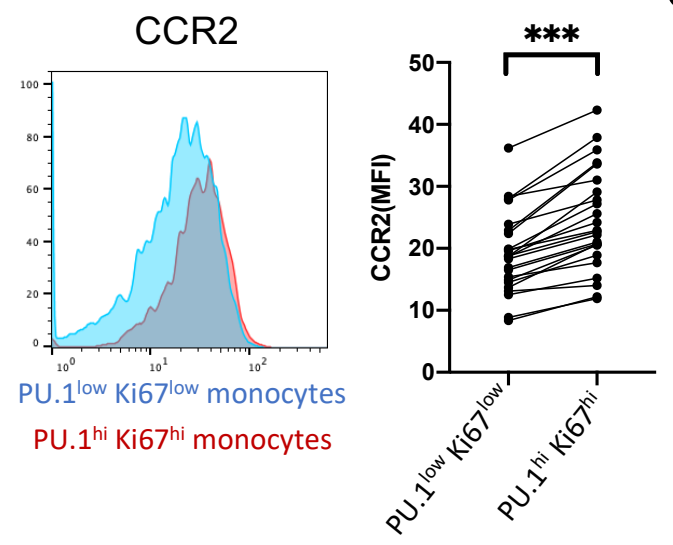

B
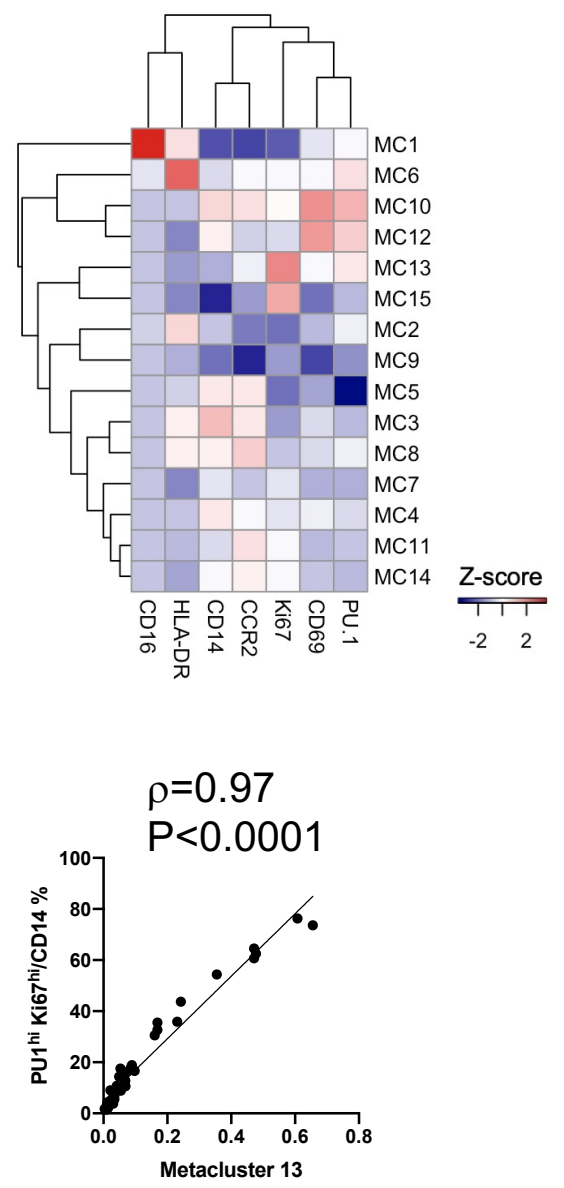

G

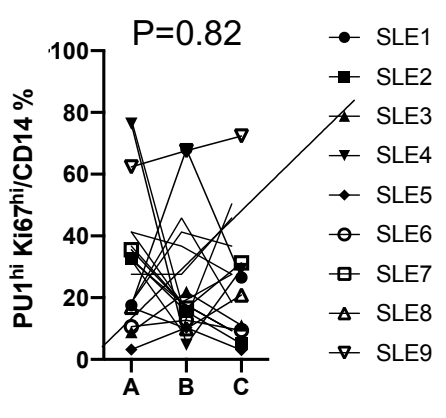


bioRxiv preprint doi: https://doi.org/10.1101/2021.11.08.467791. this version posted November 10,2021 . The copyright holder for this preprint

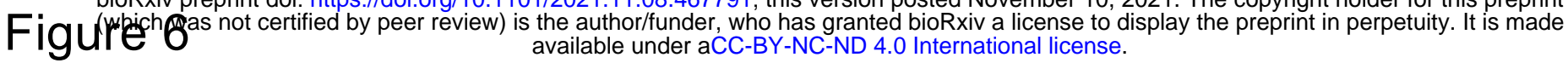

A

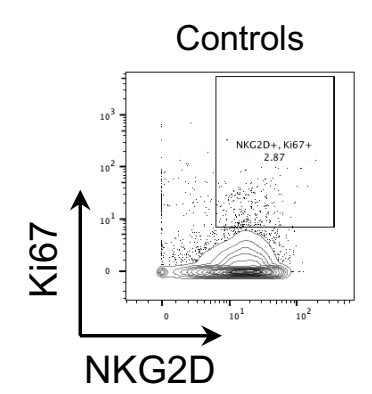

C

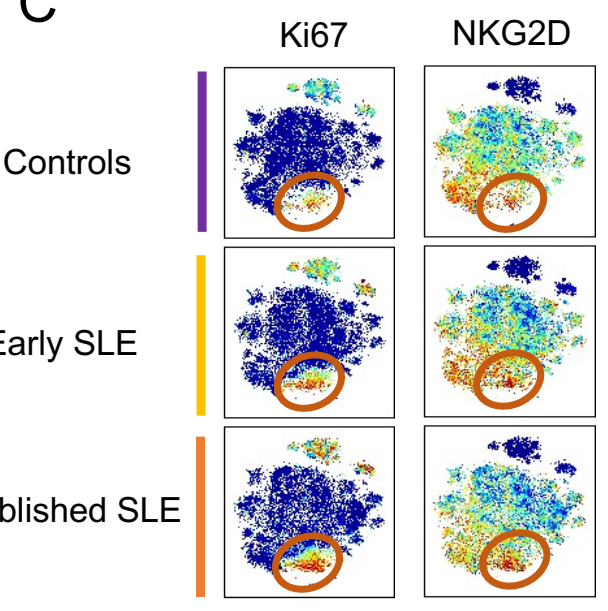

B
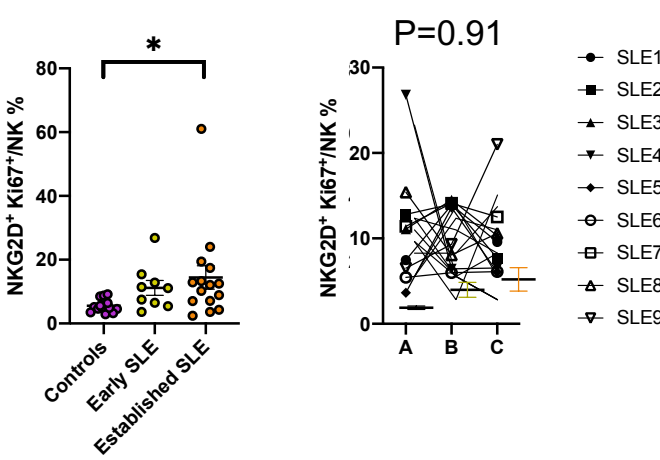

D

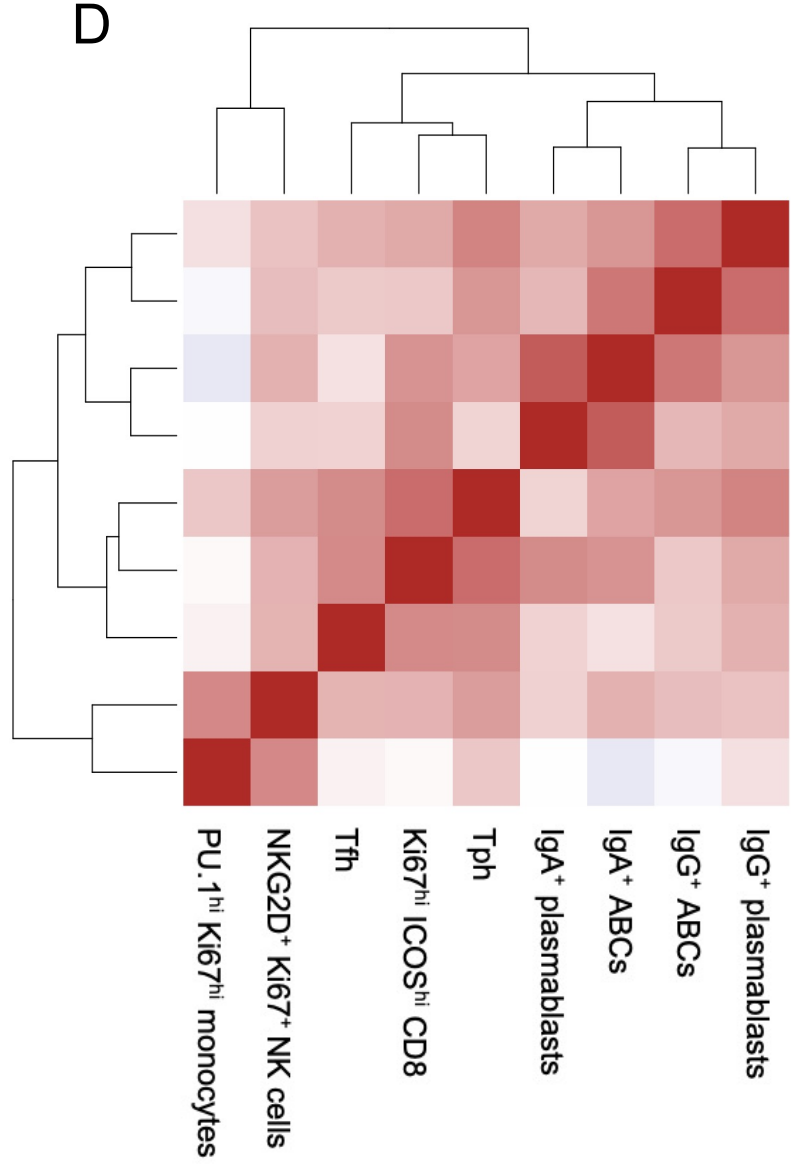

IgG ${ }^{+}$plasmablasts $\lg \mathrm{G}^{+} \mathrm{ABCs}$ $\lg \mathrm{A}^{+} \mathrm{ABCs}$ $\lg \mathrm{A}^{+}$plasmablasts Tph Ki $67^{\text {hi }} I C O S^{\text {hi }} \operatorname{CD} 8$ Tfh $\mathrm{NKG}^{2} \mathrm{D}^{+} \mathrm{Ki}^{+} 7^{+} \mathrm{NK}$ cells PU. $1^{\text {hi }}$ Ki67 $67^{\text {hi }}$ monocytes

Correlation coefficient $\begin{array}{lll}-1 & 1\end{array}$
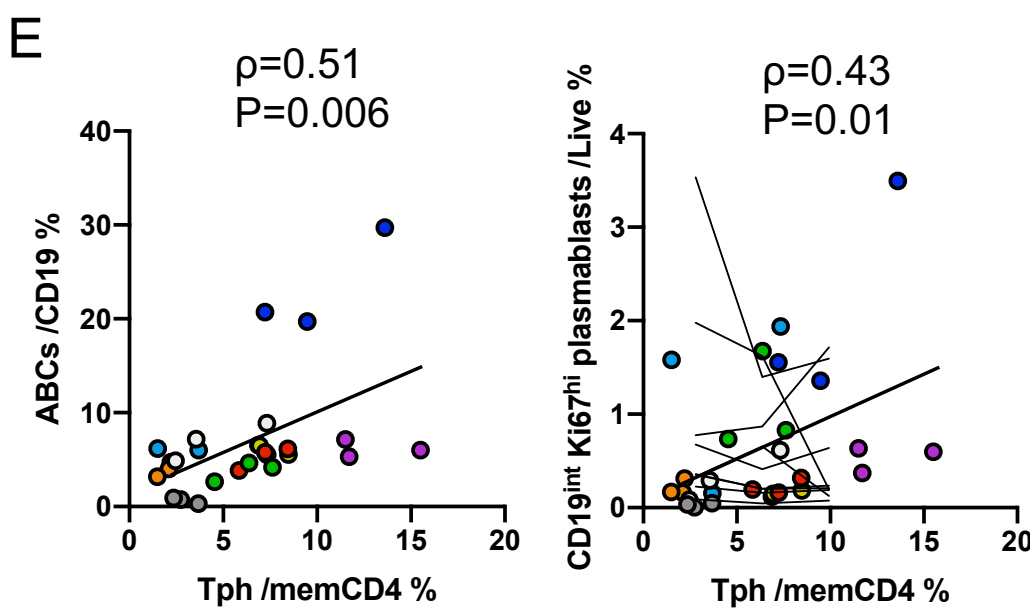

Tph /memCD4 \%
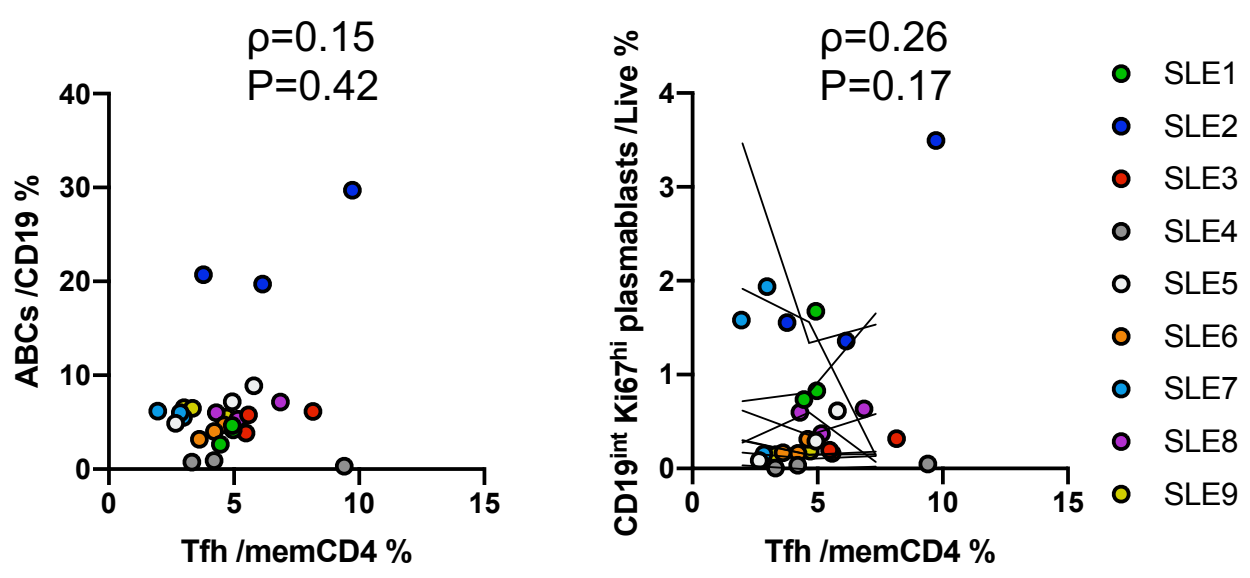
bioRxiv preprint doi: https://doi.org/10.1101/2021.11.08.467791; this version posted November 10,2021 . The copyright holder for this preprint

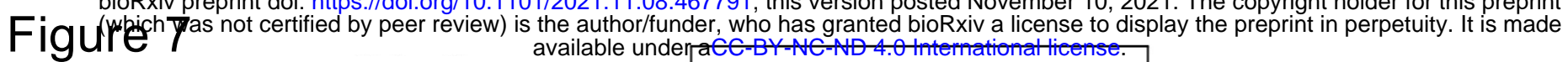

A
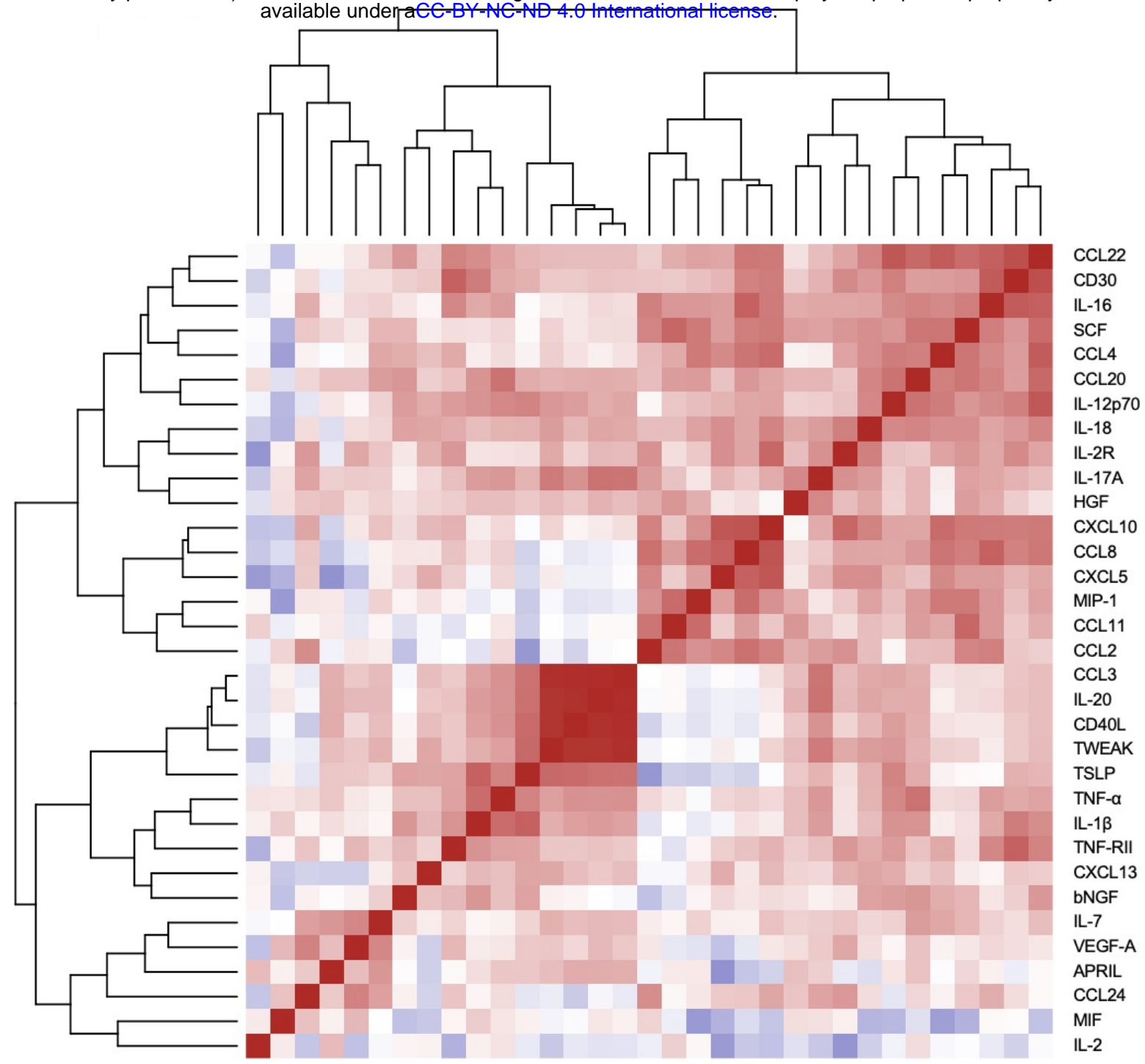

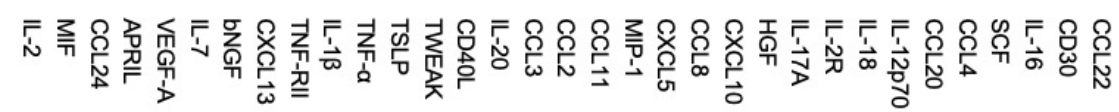

Correlation coefficient

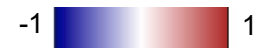
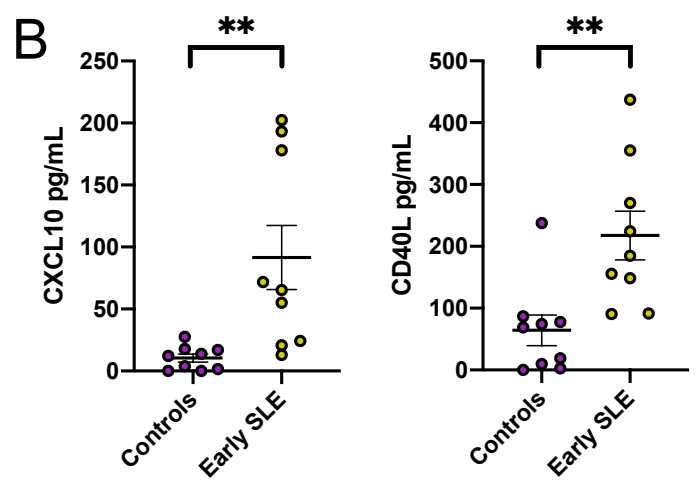

C
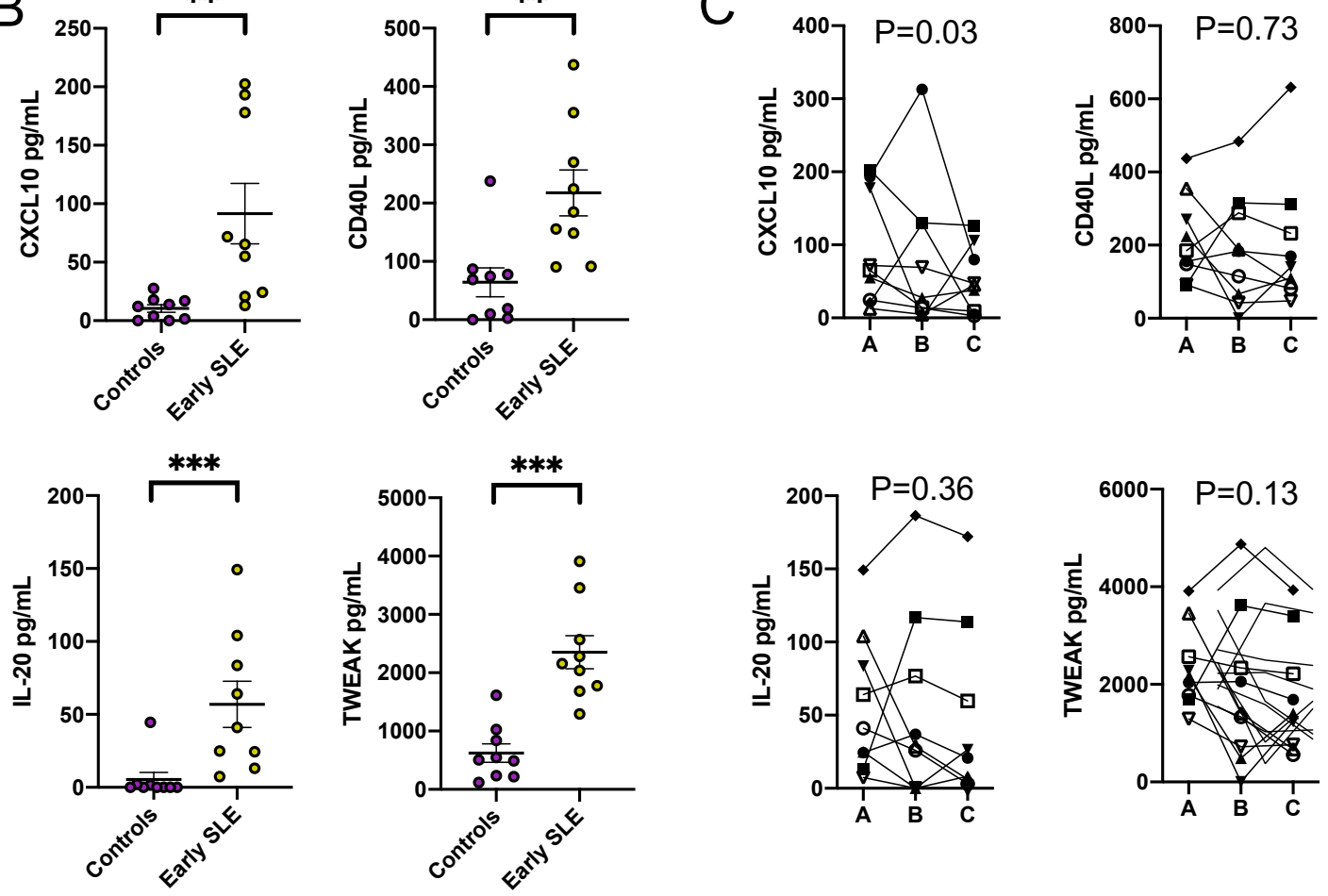

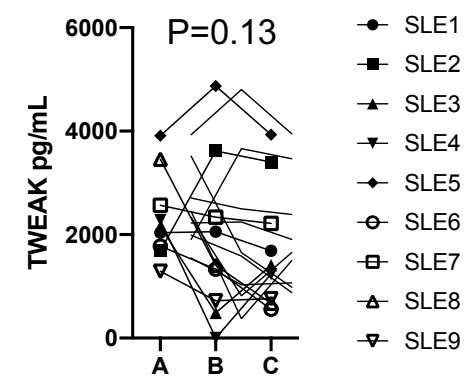

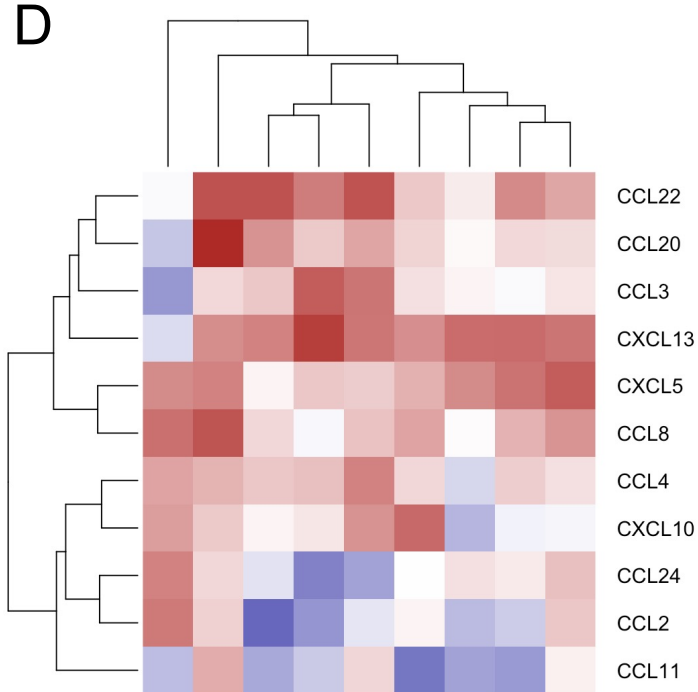

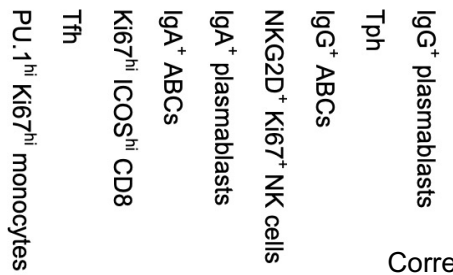

CCL22 CCL3

CXCL13 CCL8 CCL4 CXCL10 CCL2

on coefficien $- 0 . 6 \longdiv { - 1 0 . 6 }$ 
Figure 8

\section{At diagnosis}

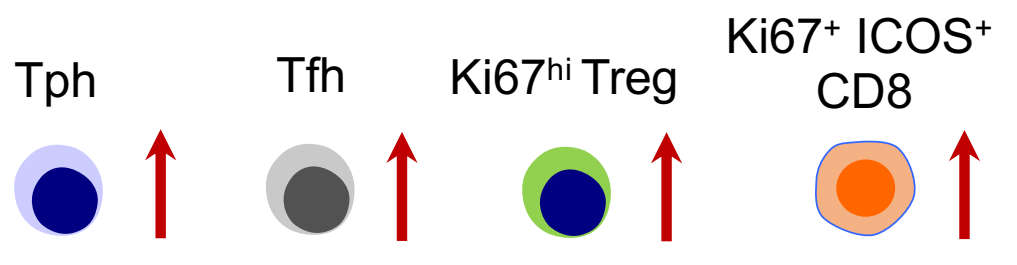

1 year after the diagnosis

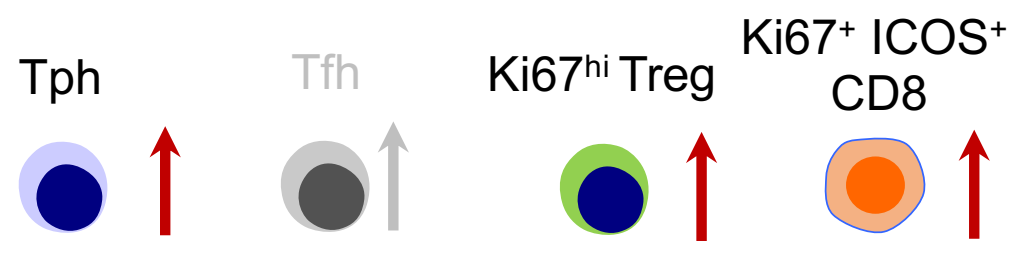

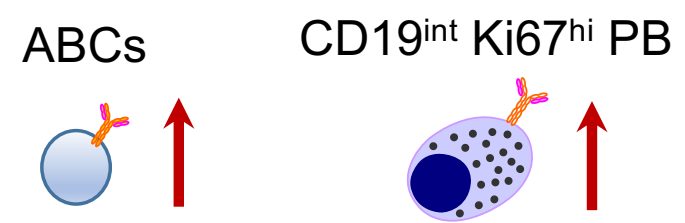

Ki67hi PU. $1^{\text {hi }}$ monocytes

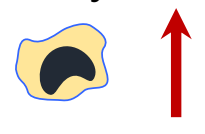

$\mathrm{NKG}^{2} \mathrm{D}^{+} \mathrm{Ki} 67^{+} \mathrm{NK}$

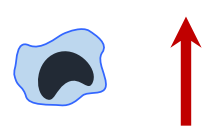

$\operatorname{CXCL10\uparrow }$

CD40L

$\mathrm{IL}-20$ 个
TWEAK $\uparrow$
Ki67 hi PU.1 $1^{\text {hi }}$ monocytes

(ब) $\mathrm{NKG} 2 \mathrm{D}^{+} \mathrm{Ki} 67^{+} \mathrm{NK}$

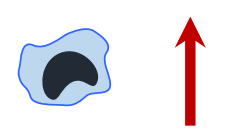

CD40L

IL-20 个 